\title{
Interaction between Dark Matter and Dark Energy and the Cosmological Coincidence Problem
}

\author{
Kourosh Nozari, Noushin Behrouz, and Narges Rashidi \\ Department of Physics, Faculty of Basic Sciences, University of Mazandaran, P.O. Box 47416-95447, Babolsar, Iran \\ Correspondence should be addressed to Kourosh Nozari; knozari@umz.ac.ir
}

Received 27 May 2014; Revised 27 October 2014; Accepted 9 November 2014; Published 2 December 2014

Academic Editor: Frank Filthaut

Copyright ( 2014 Kourosh Nozari et al. This is an open access article distributed under the Creative Commons Attribution License, which permits unrestricted use, distribution, and reproduction in any medium, provided the original work is properly cited. The publication of this article was funded by SCOAP S $^{3}$

\begin{abstract}
We consider a quintessence model of dark energy inspired by scalar-tensor theories of gravity where the scalar field is nonminimally coupled to gravity and dark matter. By considering exponential potential as self-interaction potential, the stability and existence of the critical points are discussed in details. With nonminimally coupled dark sector with gravity, we obtain scaling solutions to address the coincidence problem by considering complex velocity for dark matter. The statefinder diagnostic shows that the equation of state reaches $\Lambda C D M$ model in the future.
\end{abstract}

\section{Introduction}

Recent observational pieces of evidence [1-6] have revealed that today the energy density of the universe is dominated by a component with negative pressure that violates the strong energy condition (SEC). This yet unknown component is called "dark energy" (DE), which derives the positively accelerated phase of the universe expansion. Modification of gravity on vast scale may be another alternative to explain this accelerated phase of expansion $[7,8]$. In the framework of dark energy proposal, the simplest model is a cosmological constant, whereas, the cosmological constant proposal suffers from serious difficulties such as a huge fine-tuning [9-12]. In fact, there is no convincing explanation for the very small value of the $\Lambda$ and also no concrete solution for coincidence problem and need for huge fine-tuning. Coincidence problem is that why are the energy densities of both dark components (dark energy and dark matter) the same order of magnitude today? The dynamical dark energy models such as light scalar fields, quintessence $[13,14]$, phantom fields [15], tachyon fields $[16,17]$, and other similar theories that are capable of explaining the late-time cosmic acceleration can alleviate the coincidence problem [18]. It is possible to construct a generalized model of quintessence field that the background and the dark energy evolve independently, but there is a nonminimal coupling between both dark components
[19-26]. Because the nature of the dark matter (DM) is yet unknown, it is possible to consider additional interactions between the dark components, without tension with the observational facts. Nevertheless, solar system tests impose some restrictions on the nonminimal coupling between the dark matter and the dark energy [27]. Currently no specific coupling between the dark sectors has been known based on fundamental theories. Therefore, suggested coupling models will necessarily be phenomenological $[28,29]$, though some models seem to have more physical justification than others [19]. Here we are going to study an interacting model in which the dark sectors are coupled to each other in a background gravitational field nonminimally coupled to the dark energy. We seek scaling solutions to address the coincidence problem in a manner much similar to the chameleon mechanism.

The paper is organized as follows. In Section 2 we review some new details of two models of interaction between the dark sectors and scalar curvature with minimal and nonminimal coupling. We present existence and stability of the critical points in phase space. In Section 3, we present a model in which the scalar field is coupled nonminimally with both gravity and dark matter. The cosmological dynamics of the model is studied via dynamical system technique and also statefinder diagnostic. Scaling solutions are obtained in this section and the coincidence problem is addressed through a manner similar to the chameleon mechanism. This is done 
by relaxing the reality of sound velocity and allowing to have complex sound speed profile. The coincidence problem can be evaded in this case since, a constant ratio between the dark energy and dark matter density is a stable attractor. We suppose universal coupling of the quintessence field to all sorts of matter (radiation is excluded). We note also that actually there is no need to consider the coupling between quintessence field and baryonic matter. In fact, the quintessence field has coupling with dark matter. In Section 4, conclusions are presented.

\section{Preliminaries}

2.1. Minimal Coupling of Quintessence Field with Dark Matter. The action for a quintessence field minimally coupled with the dark matter is given by

$$
\mathcal{S}=\int d^{4} x \sqrt{-g}\left[\frac{R}{2}-\frac{1}{2} g^{a b} \nabla_{a} \varphi \nabla_{b} \varphi-V(\varphi)+\mathscr{L}_{m}\right],
$$

where $R$ is the curvature scalar, $\varphi$ is the quintessence scalar field, $V(\varphi)$ the quintessence self-interaction potential, which we consider as $V(\varphi)=V_{0} e^{-\lambda \varphi}$, where $\lambda$ and $V_{0}$ are positive constants and $\mathscr{L}_{m}$ is the Lagrangian density of matter (all sorts of matter except radiation and baryonic matter. Note that the only sort of matter here is the dark matter). The FRW line element is given by

$$
\begin{gathered}
d s^{2}=-d t^{2}+a^{2}(t) \frac{d r^{2}}{1-k r^{2}}+a^{2}(t) r^{2} d \Omega^{2}, \\
d \Omega^{2}=d \theta^{2}+\sin ^{2} \theta d \phi^{2},
\end{gathered}
$$

where $t$ is the cosmic time, $(r, \theta, \phi)$ are the spatial (radial and angular) coordinates and $k$ is the spatial curvature where $k=$ $0,+1,-1$ corresponding to the flat, closed and open universes, respectively. We consider the system of units in which $8 \pi G=$ $c=\hbar=1$. The field equations that are derived from the action (1) are

$$
\begin{gathered}
3 H^{2}+\frac{3 k}{a^{2}}=\rho_{m}+\frac{1}{2} \dot{\varphi}^{2}+V(\varphi), \\
2 \dot{H}+3 H^{2}+\frac{k}{a^{2}}=(1-\gamma) \rho_{m}-\frac{1}{2} \dot{\varphi}^{2}+V(\varphi), \\
\ddot{\varphi}+3 H \dot{\varphi}+\frac{d V(\varphi)}{d \varphi}=0,
\end{gathered}
$$

where $\gamma \equiv 1+w_{m}$ is the barotropic index which depends on the type of matter. The continuity equations are

$$
\dot{\rho}_{m}+3 H \gamma \rho_{m}=0, \quad \dot{\rho}_{\varphi}+3 H\left(1+\omega_{\varphi}\right) \rho_{\varphi}=0 .
$$

The Friedmann equation (3) with $k=0$ is as follows:

$$
1=\Omega_{m}+\Omega_{\varphi}
$$

which $\Omega_{m}=\rho_{m} / 3 H^{2}$ and $\Omega_{\varphi}=\rho_{\varphi} / 3 H^{2},(7)$ implies that

$$
0 \leq \Omega_{\varphi}, \quad \Omega_{m} \leq 1 .
$$

We study cosmological dynamics of the model by translating our equations in the language of the autonomous dynamical system. We define the following dimensionless quantities:

$$
x_{1}=\frac{\dot{\varphi}}{\sqrt{6} H}, \quad x_{2}=\frac{\sqrt{V(\varphi)}}{\sqrt{3} H}, \quad x_{3}=\frac{\sqrt{\rho_{m}}}{\sqrt{3} H} .
$$

By rewriting the Friedmann equation (3) (with $k=0$ ) in terms of the new variables, we obtain a constraint on the parameters space of the model as follows:

$$
1=x_{1}^{2}+x_{2}^{2}+x_{3}^{2}
$$

which allows to seek for evolution of just two variables since the third one can be obtained by this constraint. In what follows we choose $x_{1}$ and $x_{3}$ as our independent variables. Using these phase space variables, the evolution equations (4) and the equation of state parameter $\omega_{\text {tot }}=P_{\text {tot }} / \rho_{\text {tot }}=$ $\left(P_{\varphi}+(\gamma-1) \rho_{m}\right) /\left(\rho_{\varphi}+\rho_{m}\right)$ can be rewritten as follows:

$$
\begin{gathered}
\frac{\dot{H}}{H^{2}}=-\frac{3}{2} \gamma x_{3}^{2}-3 x_{1}^{2}, \\
\frac{\ddot{\varphi}}{H^{2}}=-3 \sqrt{6} x_{1}+3 \lambda x_{2}^{2}, \\
\omega_{\text {tot }}=2 x_{1}^{2}+\gamma x_{3}^{2}-1 .
\end{gathered}
$$

In the next step, we introduce a new time variable $N=$ $\ln a(t)$ that is related to the cosmic time through $d N=H d t$ and obtain the following autonomous system of equations:

$$
\frac{d x_{1}}{d N}=\frac{\ddot{\varphi}}{\sqrt{6} H^{2}}-x_{1} \frac{\dot{H}}{H^{2}}, \quad \frac{d x_{3}}{d N}=-\left[\frac{3}{2} \gamma+\frac{\dot{H}}{H^{2}}\right] x_{3} .
$$

The stability around the fixed points is related to the form of the eigenvalues in each critical point. In Table 1 we exhibit the properties of the critical points such as the existence and stability. Furthermore, we present $\omega_{\text {totc }}$ as the value of the total equation of state parameter at the critical points to see the possibility of accelerated expansion in this setup $\left(\omega_{\text {totc }}<-1 / 3\right)$. The eigenvalues can be obtained by using the above autonomous equations, the results of which are shown in Table 2. As we see in Table 1, among the five fixed points, only point $E_{ \pm}$exhibits scaling solution for late-time. Using (8), (9), (10), and (13), we find for this point

$$
\begin{gathered}
0<\Omega_{m}=x_{3}^{2}, \quad \Omega_{\varphi}=\left(1-x_{3}^{2}\right)<1, \\
\omega_{\text {tot }}<-\frac{1}{3} .
\end{gathered}
$$

According to Table 1, this point can be either stable node or stable spiral. In Figure 1 , if we choose $\lambda$ and $\gamma$ parameters from the blue shaded region, point $E_{ \pm}$will be stable node, but if we select these parameters from the green shaded region, point $E_{ \pm}$will be stable spiral. The phase space of this critical point is illustrated in Figure 3(a). All the phase space trajectories diverge from the unstable point (radiation 
TABLe 1: Properties of the critical points.

\begin{tabular}{|c|c|c|c|c|c|}
\hline Point $\left(x_{1 c}, x_{3 c}\right)$ & Existence & Stability & $\Omega_{\varphi}$ & $\omega_{\text {totc }}$ & $\ddot{a}_{c}>0$ \\
\hline$A(+1,0)$ & $\forall \lambda, \gamma$ & $\begin{array}{l}\text { Saddle point if } \lambda>\sqrt{6} \text { and } 0<\gamma<2 \text {, } \\
\text { unstable node if } \lambda<\sqrt{6} \text { and } 0<\gamma<2\end{array}$ & 1 & 1 & No \\
\hline$B(-1,0)$ & $\forall \lambda, \gamma$ & $\begin{array}{l}\text { Saddle point if } \lambda<-\sqrt{6} \text { and } 0<\gamma<2 \text {, } \\
\text { unstable node if } \lambda>-\sqrt{6} \text { and } 0<\gamma<2\end{array}$ & 1 & 1 & No \\
\hline$C\left(\frac{\sqrt{6} \lambda}{6}, 0\right)$ & $\forall \gamma, \lambda^{2} \leq 6$ & $\begin{array}{l}\text { Stable node if } \lambda^{2}<6 \text { and } \lambda^{2}<3 \gamma \\
\text { saddle point if } 3 \gamma<\lambda^{2}<6\end{array}$ & 1 & $\frac{\lambda^{2}}{3}-1$ & $\lambda^{2}<2$ \\
\hline$D_{ \pm}(0, \pm 1)$ & $\forall \lambda, \gamma$ & Saddle point if $0<\gamma<2$ & 0 & $\gamma-1$ & $\gamma<\frac{2}{3}$ \\
\hline$E_{ \pm}\left(\frac{\sqrt{6}}{2 \lambda} \gamma, \pm \frac{\sqrt{\lambda^{2}-3 \gamma}}{\lambda}\right.$ & $\lambda^{2} \geq 3 \gamma$ & $\begin{array}{l}\text { Stable node for left panel of Figure } 1 \\
\text { Stable spiral for right panel of Figure } 1\end{array}$ & $\frac{3 \gamma}{\lambda^{2}}$ & $\gamma-1$ & $\gamma<\frac{2}{3}$ \\
\hline
\end{tabular}

TABLE 2: The eigenvalues of the critical points.

\begin{tabular}{ll}
\hline Point $\left(x_{1 c}, x_{3 c}\right)$ & $\sigma_{1}, \sigma_{2}$ \\
\hline$A(+1,0)$ & $6-\sqrt{6} \lambda,-\frac{3}{2} \gamma+3$ \\
\hline$B(-1,0)$ & $6+\sqrt{6} \lambda,-\frac{3}{2} \gamma+3$ \\
\hline$C\left(\frac{\sqrt{6} \lambda}{6}, 0\right)$ & $\frac{1}{2} \lambda^{2}-3,+\frac{1}{2} \lambda^{2}-\frac{3}{2} \gamma$ \\
\hline$D_{ \pm}(0, \pm 1)$ & $\frac{3}{2} \gamma-3,3 \gamma$ \\
\hline$E_{ \pm}\left(\frac{\sqrt{6}}{2 \lambda} \gamma, \pm \frac{3}{\lambda^{2}-3 \gamma}\right)$ & $\frac{3}{4}\left(\gamma \lambda-2 \lambda+\frac{\sqrt{9 \gamma^{2} \lambda^{2}-24 \gamma^{3}-20 \gamma \lambda^{2}+48 \gamma^{2}+4 \lambda^{2}}}{\lambda}\right)$, \\
\hline
\end{tabular}

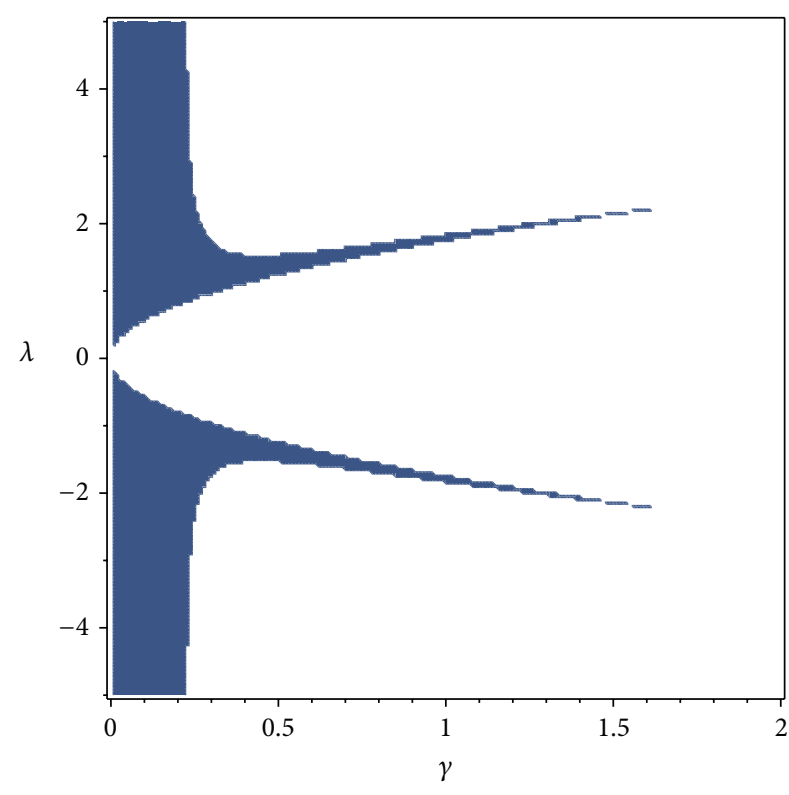

(a)

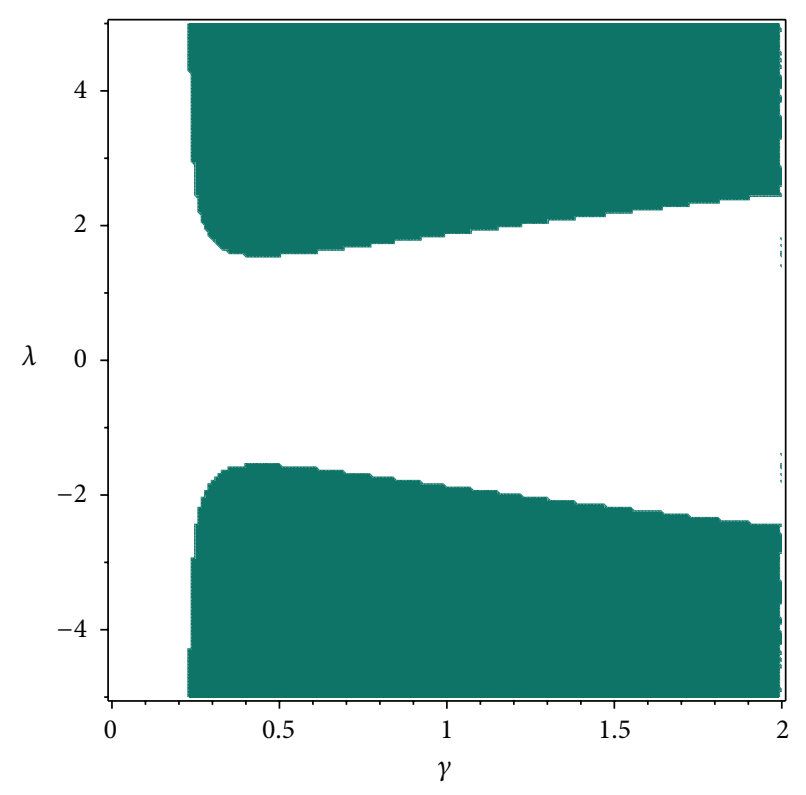

(b)

FIgURE 1: $E_{ \pm}$is a stable node in the blue shaded region of $\lambda-\gamma$ space (a), while it is a stable spiral in the green shaded region of (b). 
domination) and converge towards the attractor. As has been shown in Figure 4(a), this critical point cannot cross the phantom divide line and reaches to recent quantity of $\omega$ that tends to be less than -1 [30]. To have such an observationally supported crossing, we add a further ingredient to the theory: the nonminimal coupling between the dark energy and scalar curvature [31]. Furthermore, it is important to keep in mind that if we consider negative values of $\gamma$ for $E_{ \pm}$to have $\omega_{\text {totc }}<$ -1 , then the critical point $D_{ \pm}$will be also a stable attractor point, which is not acceptable since it should be a transient matter dominated epoch.

2.2. Nonminimal Coupling of Dark Energy with Ricci Scalar. We consider the following action in the spirit of the scalartensor theories:

$$
\begin{aligned}
\mathcal{S}=\int & d^{4} X \sqrt{-g} \\
& \times\left[\left(\frac{1}{2}-\frac{1}{2} \xi \varphi^{2}\right) R-\frac{1}{2} g^{a b} \nabla_{a} \varphi \nabla_{b} \varphi-V(\varphi)+\mathscr{L}_{m}\right],
\end{aligned}
$$

where $\xi$ is the nonminimal coupling between the quintessence field as dark energy and scalar curvature. The energy density and pressure of the scalar field nonminimally coupled to gravity are defined as follows [32]:

$$
\begin{aligned}
\rho_{\varphi}= & \frac{1}{2} \dot{\varphi}^{2}+V(\varphi)+3 \xi H^{2} \varphi^{2}+6 \xi H \varphi \dot{\varphi}, \\
p_{\varphi}= & \frac{1}{2} \dot{\varphi}^{2}-V(\varphi)-4 \xi H \varphi \dot{\varphi}-2 \xi \dot{\varphi}^{2}-2 \xi \varphi \ddot{\varphi} \\
& -2 \xi \dot{H} \varphi^{2}-3 \xi H^{2} \varphi^{2} .
\end{aligned}
$$

The field equations derived from action (16) are given as

$$
\begin{aligned}
3 H^{2}+\frac{3 k}{a^{2}}=\rho_{m}+ & \frac{1}{2} \dot{\varphi}^{2}+3 \xi H^{2} \varphi^{2}+6 \xi H \varphi \dot{\varphi}, \\
2 \dot{H}+3 H^{2}+\frac{k}{a^{2}}= & (1-\gamma) \rho_{m}-\frac{1}{2} \dot{\varphi}^{2}+V(\varphi) \\
& +4 \xi H \varphi \dot{\varphi}-2 \xi \dot{\varphi}^{2}+2 \xi \varphi \ddot{\varphi} \\
& +2 \xi \dot{H} \varphi^{2}+3 \xi H^{2} \varphi^{2}, \\
\ddot{\varphi}+3 H \dot{\varphi}+ & \frac{d V(\varphi)}{d \varphi}+\xi R \varphi=0,
\end{aligned}
$$

where $\gamma \equiv 1+\omega_{m}$ is the barotropic index of ordinary matter. The continuity equations are given as

$$
\dot{\rho}_{m}+3 H \gamma \rho_{m}=0, \quad \dot{\rho}_{\varphi}+3 H\left(1+\omega_{\varphi}\right) \rho_{\varphi}=0 .
$$

Similar to the previous subsection, (7) and (8) are valid for this case too. The dimensionless quantities for this case are $x_{1}, x_{2}$, and $x_{3}$ defined in the previous subsection, plus the additional one defined as follows:

$$
x_{4}=\sqrt{\xi} \varphi \text {. }
$$

From Friedmann equation (18) with zero spatial curvature $(k=0)$, we have the following constraint:

$$
1=x_{1}^{2}+x_{2}^{2}+x_{3}^{2}+x_{4}^{2}+2 \sqrt{6 \xi} x_{1} x_{4} \text {. }
$$

In terms of these new variables, the acceleration equation (19), the scalar field's equation of motion (20), and equation of state parameter are given by the following expressions:

$$
\begin{gathered}
\frac{\dot{H}}{H^{2}}=\left(-\frac{3}{2} \gamma x_{3}^{2}-3 x_{1}^{2}+6 \xi x_{1}^{2}-4 \sqrt{6 \xi} x_{1} x_{4}\right. \\
\left.+3 \lambda \sqrt{\xi} x_{4} x_{2}^{2}-12 \xi x_{4}^{2}\right) \\
\times\left(1-x_{4}^{2}+6 \xi x_{4}^{2}\right)^{-1}, \\
\frac{\ddot{\varphi}}{H^{2}}=-3 \sqrt{6} x_{1}+3 \lambda x_{2}^{2}-12 \sqrt{\xi} x_{4}-6 \sqrt{\xi} x_{4} \frac{\dot{H}}{H^{2}}, \\
\omega_{\text {tot }}=(2-4 \xi) x_{1}^{2}+\gamma x_{3}^{2}+\frac{2}{3} \sqrt{6 \xi} x_{1} x_{4}-\frac{2}{3} \sqrt{\xi} x_{4} \frac{\dot{H}}{H^{2}} \\
-\frac{2}{3} x_{4}^{2} \frac{\ddot{\varphi}}{H^{2}}-1 .
\end{gathered}
$$

We differentiate the phase space dimensionless variables with respect to $N=\ln a(t)$ to find

$$
\begin{gathered}
\frac{d x_{1}}{d N}=\frac{\sqrt{6}}{6} \frac{\ddot{\varphi}}{H^{2}}-x_{1} \frac{\dot{H}}{H^{2}}, \quad \frac{d x_{3}}{d N}=-\left[\frac{3}{2} \gamma+\frac{\dot{H}}{H^{2}}\right] x_{3}, \\
\frac{d x_{4}}{d N}=\sqrt{6 \xi} x_{1} .
\end{gathered}
$$

The critical points and their stability around the fixed points are shown in Table 3 (for $0<\gamma<2$ ) and eigenvalues of the Jacobian matrix are presented in Table 4. As Table 3 shows, there is no critical point for late-time. Attractor critical points $B_{+}$and $B_{-}$belong to the future. Fixed point $B_{+}$can be either a stable node or a stable spiral depending on the values of $\lambda$ and $\xi$ from blue shaded region or green shaded region in Figure 2. However, critical point $B_{-}$is stable spiral. The 2dimensional phase space of this critical point is depicted in the right panel of Figure 3. So, we see that the nonminimal model allows to reach a stable phase in the future with equation of state that tends to the $\triangle C D M$ one in the future. The behavior of $\omega_{\text {totc }}$ is shown in Figure $4(\mathrm{~b})$. There is no crossing of the phantom divide even in this nonminimal model. So, similar to the minimal model, this nonminimal model is not a suitable framework for late time cosmic dynamics and it is not capable of addressing the coincidence problem.

\section{Dark Energy Coupled Nonminimally with Gravity and Dark Matter}

We consider a scalar field, as dark energy component, which is nonminimally coupled to gravity and dark matter in the spirit of scalar-tensor theories:

$$
\begin{aligned}
\mathcal{S}=\int & d^{4} X \sqrt{-g} \\
& \times\left[\left(\frac{1}{2}-\frac{1}{2} \xi \varphi^{2}\right) R-\frac{1}{2} g^{a b} \nabla_{a} \varphi \nabla_{b} \varphi-V(\varphi)+\alpha \varphi^{2} \mathscr{L}_{m}\right],
\end{aligned}
$$




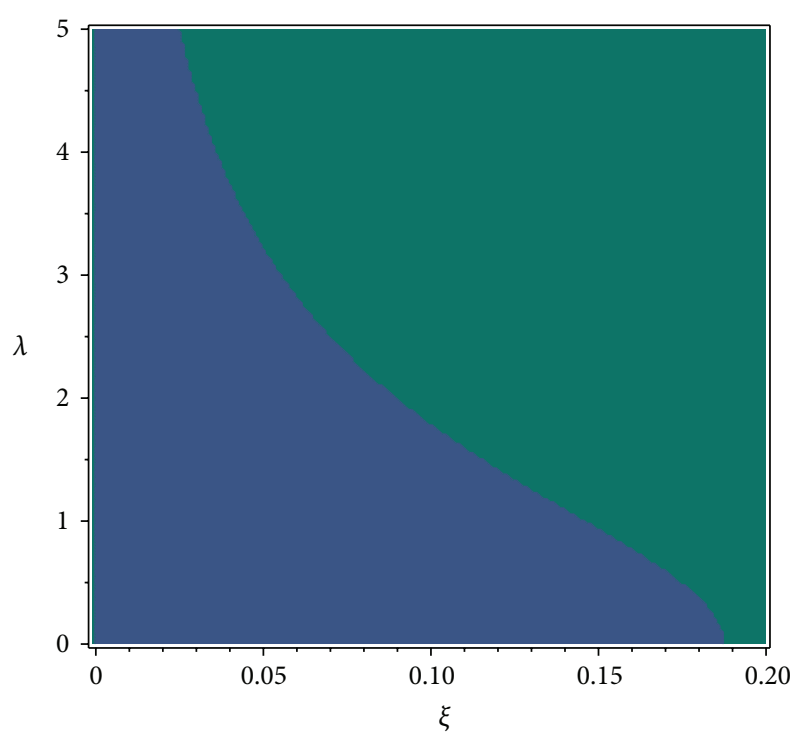

Stable node

Stable spiral

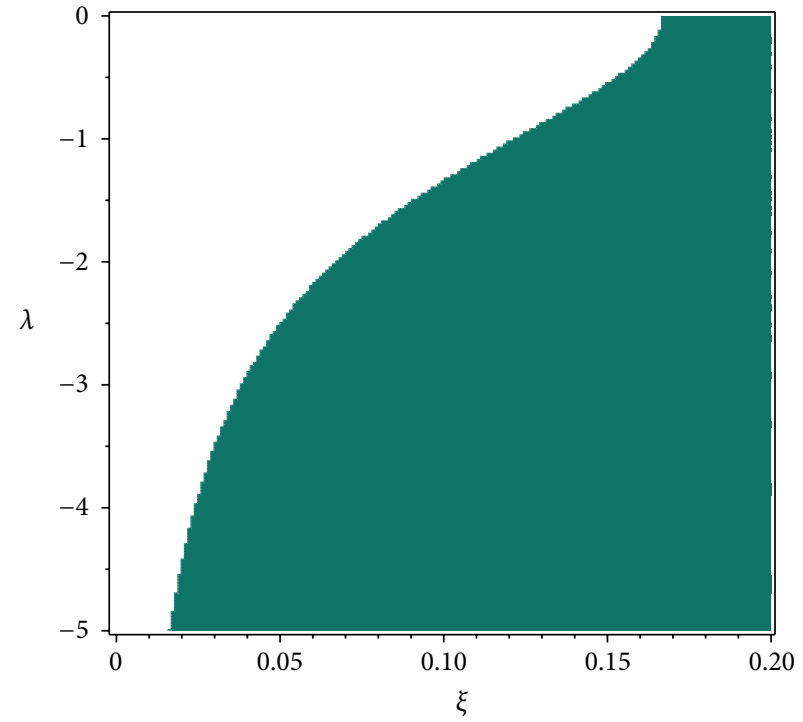

Stable spiral

(a)

(b)

Figure 2: (a) In the $\lambda$ and $\xi$ parameters space the blue shaded region is valid for stable nodes and the green shaded one is valid for stable spirals for critical point $B_{+}$. (b) The green shaded region for stable spiral in the $\lambda$ and $\xi$ parameters space for critical point $B_{-}$.

TABLE 3: The properties of the critical points.

\begin{tabular}{|c|c|c|c|c|c|}
\hline Point $\left(x_{1 c}, x_{3 c}, x_{4 c}\right)$ & Existence & Stability & $\Omega_{\varphi}$ & $\omega_{\text {tot }}$ & $\ddot{a}>0$ \\
\hline$A(0,0,1)$ & $\forall \lambda, \xi, \gamma$ & Saddle point & 1 & $\frac{1}{3}$ & No \\
\hline$B_{+}\left(0,0, \frac{-2 \sqrt{\xi}+\sqrt{\lambda^{2}+4 \xi}}{\lambda}\right)$ & $\forall \gamma, \lambda>0, \xi>0$ & $\begin{array}{l}\text { Stable node for left panel of Figure } 2 \\
\text { (blue shaded region) } \\
\text { stable spiral for left panel of Figure } 2 \\
\text { (green shaded region) }\end{array}$ & 1 & -1 & 1 \\
\hline$B_{-}\left(0,0, \frac{-2 \sqrt{\xi}-\sqrt{\lambda^{2}+4 \xi}}{\lambda}\right)$ & $\forall \gamma, \lambda<0, \xi>0$ & Stable spiral for right panel of Figure 2 & 1 & -1 & 1 \\
\hline$C_{ \pm}(0, \pm 1,0)$ & $\forall \lambda, \xi, \gamma$ & Saddle point & 0 & $\gamma-1$ & $\gamma<\frac{2}{3}$ \\
\hline
\end{tabular}

TABLE 4: The eigenvalues of the critical points.

\begin{tabular}{ll}
\hline Point $\left(x_{1 c}, x_{3 c}, x_{4 c}\right)$ & $\sigma_{1}, \sigma_{2}, \sigma_{3}$ \\
\hline$A(0,0,1)$ & $-1,4,2-\frac{3}{2} \gamma$ \\
\hline$B_{+}\left(0,0, \frac{-2 \sqrt{\xi}+\sqrt{\lambda^{2}+4 \xi}}{\lambda}\right)$ & $-\frac{3}{2} \gamma, G_{1}, G_{2}$ \\
\hline$B_{-}\left(0,0, \frac{-2 \sqrt{\xi}-\sqrt{\lambda^{2}+4 \xi}}{\lambda}\right)$ & $-\frac{3}{2} \gamma, G_{3}, G_{4}$ \\
\hline$C_{ \pm}(0, \pm 1,0)$ & $3 \gamma, \frac{3}{4} \gamma-\frac{3}{2}+\frac{1}{4} \sqrt{9 \gamma^{2}+144 \gamma \xi-36 \gamma-192 \gamma+36,}$ \\
\hline
\end{tabular}

where $\alpha \varphi^{2}$ is the quintessence-matter coupling function, ( $\alpha$ is constant). The energy density and pressure of the nonminimally coupled scalar field in this case are given by (17) but now with redefined $\rho_{m}$ (see (32) and (35) below).
The field equations that are derived from the action (28) with redefined $\rho_{m}$ are as follows:

$$
3 H^{2}+\frac{3 k}{a^{2}}=\rho_{m}+\frac{1}{2} \dot{\varphi}^{2}+3 \xi H^{2} \varphi^{2}+6 \xi H \varphi \dot{\varphi},
$$




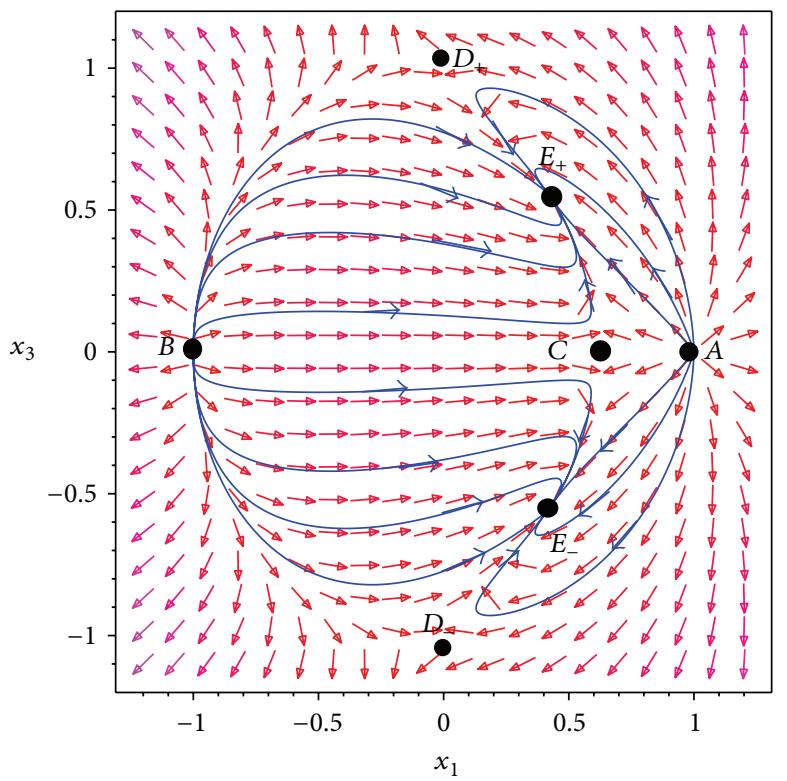

(a)

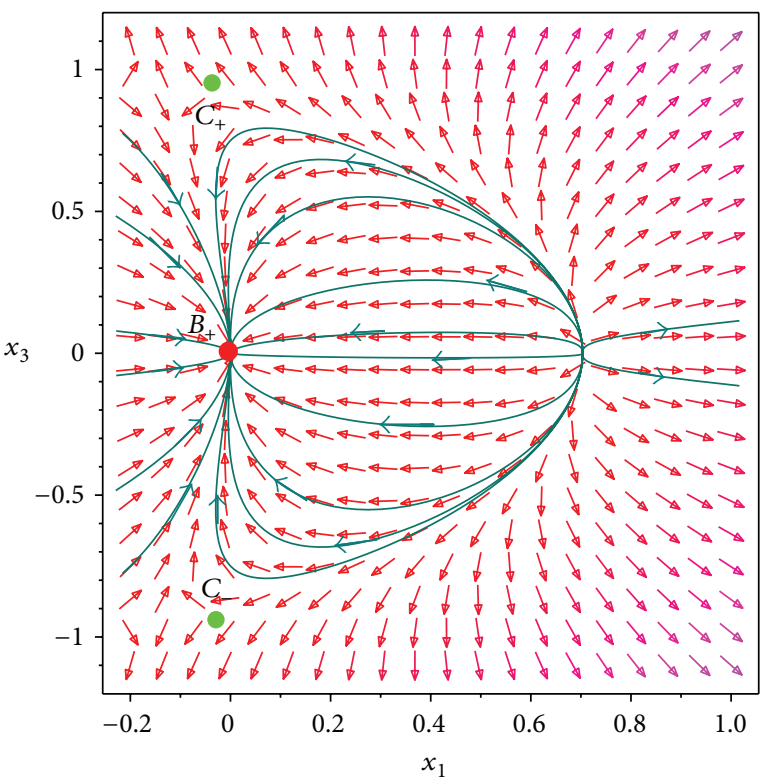

(b)

Figure 3: (a) The phase plane for critical $E_{ \pm}$(for minimal case) with $\gamma=0.5$ and $\lambda=1.48$. The critical points $E_{ \pm}(0.17,0.56)$ are stable nodes (sinks) so that the quintessence dominated solution is the late time attractor. The critical points $D_{ \pm}(0, \pm 1)$ and $C(0.6,0)$ are saddle points, but $A(+1,0), B(-1,0)$ fixed points are repeller nodes. All the phase space trajectories diverge from the unstable point and converge towards the attractor. (b) The phase plane for the critical point $B_{+}$(for nonminimal case) with $\gamma=1, \lambda=1$ and $\xi=1 / 6$. The critical point $B_{+}(0,0)$ is a stable node (a sink) so that the quintessence dominated solution is a future attractor.

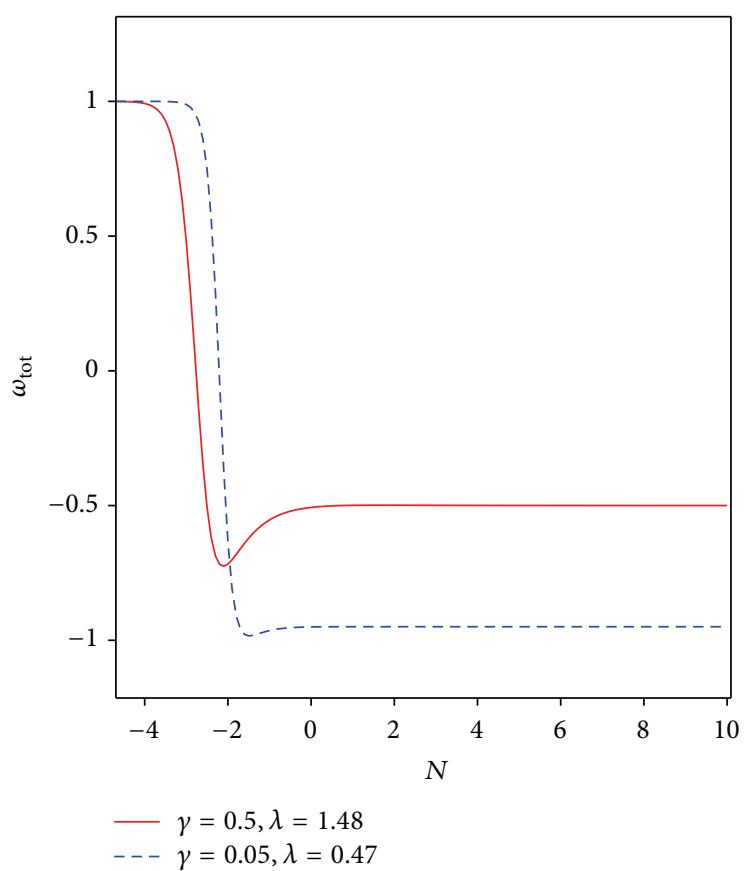

(a)

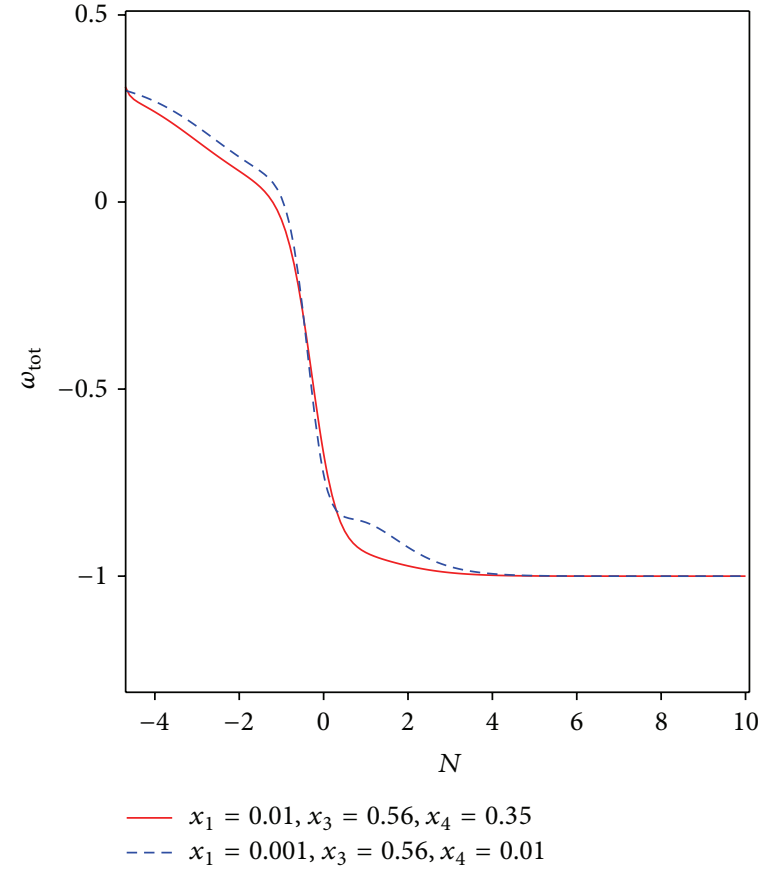

(b)

FIGURE 4: (a) Effective equation of state parameter (minimal case) versus the cosmic time for the critical points $E_{ \pm}$with initial values $x_{1}=0.41$ and $x_{3}=0.56$ and the values of $\gamma$ and $\lambda$ as given in the figure. (b) Effective equation of state parameter versus the cosmic time for the critical point $B_{+}$with $\gamma=1, \lambda=1, \xi=1 / 6$ and initial values as given in the figure. 


$$
\begin{aligned}
2 \dot{H}+3 H^{2}+\frac{k}{a^{2}}= & (1-\gamma) \rho_{m}-\frac{1}{2} \dot{\varphi}^{2}+V(\varphi)+4 \xi H \varphi \dot{\varphi} \\
& -2 \xi \dot{\varphi}^{2}+2 \xi \varphi \ddot{\varphi}+2 \xi \dot{H} \varphi^{2}+3 \xi H^{2} \varphi^{2} \\
\ddot{\varphi}+3 H \dot{\varphi} & +\frac{d V(\varphi)}{d \varphi}+\xi R \varphi=2 \alpha \varphi \rho_{m} .
\end{aligned}
$$

A general coupling between a quintessence field and the dark matter may be described by the continuity equations as

$$
\begin{gathered}
\dot{\rho}_{m}+3 H \gamma \rho_{m}=-Q, \\
\dot{\rho}_{\varphi}+3 H\left(1+\omega_{\varphi}\right) \rho_{\varphi}=Q .
\end{gathered}
$$

Here $Q=2 \alpha \varphi \dot{\varphi} \rho_{m}$ is the rate of energy density exchange in the dark sector with the following properties:

$$
\begin{aligned}
& Q>0, \quad \text { Energy transfers from dark matter } \\
& \text { to dark energy, } \\
& Q<0, \quad \text { Energy transfers from dark energy } \\
& \text { to dark matter. }
\end{aligned}
$$

After integration of (32) we find

$$
\rho_{m}=M a^{-3} e^{-\alpha \varphi^{2}}
$$

where $M$ is a constant of integration. By defining $\omega_{\text {tot }}=$ $P_{\text {tot }} / \rho_{\text {tot }}=\left(P_{\varphi}+P_{m}\right) /\left(\rho_{\varphi}+\rho_{m}\right)$ now the following equation is valid:

$$
\dot{\rho}_{\text {tot }}+3 H\left(1+\omega_{\text {tot }}\right) \rho_{\text {tot }}=0 \text {. }
$$

Like the previous subsection, (7), (8) are valid for this case too. Now we study cosmological dynamics of this interacting model as a dynamical system in phase space. Our dimensionless parameters and the Friedmann constraint are similar to (9), (22), and (23). We rewrite other important equations of the setup in terms of the new variables. The acceleration equation (30) and the equation of motion (31) in terms of the new variables take the following form, respectively:

$$
\begin{aligned}
& \frac{\dot{H}}{H^{2}}=(-\frac{3}{2} x_{3}^{2}-3 x_{1}^{2}+6 \xi x_{1}^{2}-4 \sqrt{6 \xi} x_{1} x_{4} \\
&\left.+6 \alpha x_{3}^{2} x_{4}^{2}+3 \lambda \sqrt{\xi} x_{4} x_{2}^{2}-12 \xi x_{4}^{2}\right) \\
& \times\left(1-x_{4}^{2}+6 \xi x_{4}^{2}\right)^{-1}, \\
& \frac{\ddot{\varphi}}{H^{2}}=\frac{6 \alpha x_{3}^{2} x_{4}}{\sqrt{\xi}}-3 \sqrt{6} x_{1}+3 \lambda x_{2}^{2}-12 \sqrt{\xi} x_{4}-6 \sqrt{\xi} x_{4} \frac{\dot{H}}{H^{2}} .
\end{aligned}
$$

With $N=\ln a(t)$, the following autonomous system of equations is obtained:

$$
\begin{gathered}
\frac{d x_{1}}{d N}=\frac{\sqrt{6} \alpha x_{3}^{2} x_{4}}{\sqrt{\xi}}-3 x_{1}+\frac{\sqrt{6}}{2} \lambda x_{2}^{2}-2 \sqrt{6 \xi} x_{4} \\
-2\left[\sqrt{6 \xi} x_{4}+x_{1}\right] \frac{\dot{H}}{H^{2}}, \\
\frac{d x_{3}}{d N}=-\left[\frac{\sqrt{6} \alpha x_{1} x_{4}}{\xi}+\frac{3}{2}+\frac{\dot{H}}{H^{2}}\right] x_{3}, \quad \frac{d x_{4}}{d N}=\sqrt{6 \xi} x_{1} .
\end{gathered}
$$

The critical points and stability around these points are presented in Table 5 and their eigenvalues of the Jacobian matrix are shown in Table 6.

(i) Critical Point A. The critical point $A$ behaves like a saddle point in the phase space and in this case we have no latetime acceleration. For this point the universe is radiation dominated.

(ii) Critical Point $B_{ \pm}$. Points $B_{ \pm}$represent a cosmological constant dominated accelerating phase. In this case, either a scalar field's kinetic energy term or a potential energy term plays the role of a cosmological constant. So, with a quintessence scalar field nonminimally coupled with dark matter and curvature, it is possible to realize a stable, de Sitter, future accelerating phase. The complicated stability conditions for the critical point $B_{+}$were confirmed numerically, and the results are shown in Figure 2. As we see from Figure 2(a), if we choose $\lambda$ and $\xi$ quantities from the blue shaded region, then we have a stable node, but if we select these two quantities from the green region, we will have a stable spiral. For critical point $B_{-}$there is only a stable spiral solution that is illustrated in Figure 2(b). The phase portrait for this case is shown in Figure 7(a). The behavior of $\omega_{\text {tot }}$ is drawn in Figure 8(a). This figure shows that we will reach a stable phase in the future with equation of state parameter that tends to $\triangle C D M$ model.

(iii) Critical Point $C_{ \pm}$. For this case, there is no contribution of the scalar field and the universe is dominated by matter fields other than the quintessence scalar field. These two critical points behave like saddle points in the phase space if we consider $0<\gamma<2$. For $\gamma<2 / 3$, one can obtain an accelerating phase of expansion, but this phase is not stable. However, if we consider $\gamma<0$, we will have two kinds of stable attractors. It will be a stable node if we choose $\gamma$ and $\alpha$ from the Figure 5(a), and a stable spiral if we choose $\gamma$ and $\alpha$ from Figure 5(b).

(iv) Critical Point $D_{ \pm}$. The stability conditions for critical points $D_{ \pm}$are very complicated and therefore we do not present their functional form here. However, for completeness the results are illustrated in Figure 6. There are two situations depending on the values of $\gamma$. If we consider $0<$ $\gamma<2$, there is a saddle point. On the other hand for $\gamma<$ 0 , we can consider the possibility of having complex sound speed for nonordinary matter fields. In this situation there 
TABLE 5: Properties of the critical points.

\begin{tabular}{|c|c|c|c|c|c|}
\hline Point $\left(x_{1 c}, x_{3 c}, x_{4 c}\right)$ & Existence & Stability & $\Omega_{\varphi}$ & $\omega_{\text {tot }}$ & $\ddot{a}>0$ \\
\hline$A(0,0,1)$ & $\forall \lambda, \alpha, \xi, \gamma$ & Saddle point & 1 & $\frac{1}{3}$ & No \\
\hline$B_{+}\left(0,0, \frac{-2 \xi+\sqrt{\xi \lambda^{2}+4 \xi^{2}}}{\lambda \sqrt{\xi}}\right)$ & $\begin{array}{c}\forall \alpha, \gamma \\
\lambda>0, \xi>0\end{array}$ & $\begin{array}{l}\text { Stable node for left panel of Figure } 2 \\
\text { with } 0<\gamma<2 \\
\text { (blue shaded region) } \\
\text { stable spiral for left panel of Figure } 2 \\
\text { with } 0<\gamma<2 \\
\text { (green shaded region) }\end{array}$ & 1 & -1 & 1 \\
\hline$B_{-}\left(0,0, \frac{-2 \xi-\sqrt{\xi \lambda^{2}+4 \xi^{2}}}{\lambda \sqrt{\xi}}\right)$ & $\begin{array}{c}\forall \alpha, \gamma \\
\lambda<0, \xi>0\end{array}$ & $\begin{array}{l}\text { Stable spiral for right panel of } \\
\text { Figure } 2 \text { with } 0<\gamma<2\end{array}$ & 1 & -1 & 1 \\
\hline$C_{ \pm}(0, \pm 1,0)$ & $\forall \lambda, \alpha, \xi, \gamma$ & $\begin{array}{l}\text { Saddle point if } 0<\gamma<2 \text { stable node } \\
\text { for left panel of Figure } 5 \text { with } \gamma<0 \\
\text { stable spiral for right panel of } \\
\text { Figure } 5 \text { with } \gamma<0\end{array}$ & 0 & $\gamma-1$ & $\gamma<\frac{2}{3}$ \\
\hline$D_{ \pm}\left(0, \pm \frac{\sqrt{2 \xi(4-3 \gamma)}}{2 \sqrt{\alpha}}, \sqrt{\frac{3 \gamma \xi-9 \xi+2 \alpha}{2 \alpha}}\right.$ & $0 \leq \frac{(-3 \gamma+4) \xi}{\alpha} \leq 2$ & $\begin{array}{l}\text { Saddle point if } 0<\gamma<2 \\
\text { stable node for left panel of Figure } 6 \\
\text { with } \gamma<0 \\
\text { stable spiral for right panel of } \\
\text { Figure } 6 \text { with } \gamma<0\end{array}$ & $1+\frac{(3 \gamma-4) \xi}{2 \alpha}$ & $\gamma-1$ & $\gamma<\frac{2}{3}$ \\
\hline
\end{tabular}

TABLE 6: The eigenvalues of the critical points.

\begin{tabular}{ll}
\hline Point $\left(x_{1 c}, x_{3 c}, x_{4 c}\right)$ & $\sigma_{1}, \sigma_{2}, \sigma_{3}$ \\
\hline$A(0,0,1)$ & $-1,4,2-\frac{3}{2} \gamma$ \\
\hline$B_{+}\left(0,0, \frac{-2 \xi+\sqrt{\xi \lambda^{2}+4 \xi^{2}}}{\lambda \sqrt{\xi}}\right)$ & $-\frac{3}{2} \gamma, G_{1}, G_{2}$ \\
\hline$B_{-}\left(0,0, \frac{-2 \xi-\sqrt{\xi \lambda^{2}+4 \xi^{2}}}{\lambda \sqrt{\xi}}\right)$ & $-\frac{3}{2} \gamma, G_{3}, G_{4}$ \\
\hline$C_{ \pm}(0, \pm 1,0)$ & $3 \gamma,\left(\frac{3}{4} \gamma-\frac{3}{2}+\frac{1}{4} \sqrt{9 \gamma^{2}+144 \gamma \xi-36 \gamma-192 \gamma+96 \alpha+36}\right)$, \\
\hline$D_{ \pm}\left(0, \pm \frac{\sqrt{2 \xi(4-3 \gamma)}}{2 \sqrt{\alpha}}, \sqrt{\frac{3 \gamma \xi-9 \xi+2 \alpha}{2 \alpha}}\right)$ & $\left(\frac{3}{4} \gamma-\frac{3}{2}-\frac{1}{4} \sqrt{9 \gamma^{2}+144 \gamma \xi-36 \gamma-192 \gamma+96 \alpha+36}\right)$ \\
\hline
\end{tabular}

is a stable node for values of $\lambda$ and $\alpha$ that belong to the shaded region in Figure 6(a), and a stable spiral for values of $\lambda$ and $\alpha$ belonging to the shaded region in Figure 6(b). This point is an accelerated scaling solution. Now, by considering (8), (9), (22), (23), and (26), the coincidence problem can be addressed naturally. In fact, with $0<\Omega_{m}<1$ and $0<\Omega_{\varphi}<1$, our analysis confirms that $\Omega_{m} / \Omega_{\varphi}<1$ and $\omega_{\text {tot }}<-1 / 3$. The phase portrait for this case is shown in Figure 7(b). It has been shown that if we select $\lambda$ and $\alpha$ from Figure 6 , the critical points $C_{ \pm}$(matter dominated phase) will be a saddle point and all the trajectories converge to the attractor points $D_{+}$. By adopting suitable values of quantities $\gamma, \lambda$, and $\alpha$, the current value of the dark matter density, $\Omega_{m}$, can be in agreement with recent data from Planck + WMAP [30], $\Omega_{m}=0.315_{-0.018}^{+0.016}$. The behavior of $\omega_{\text {tot }}$ is shown in Figure $8(\mathrm{~b})$. Now the total equation of state parameter crosses the phantom divide line and the universe reaches a stable phase. From Figure 8, we see that if we choose suitable values for $\gamma, \lambda$, and $\alpha$, the value of the equation of state parameter can be in agreement with recent observational data; $\omega_{\text {tot }}=-1.13_{-0.25}^{+0.23}$ according to the Planck $+\mathrm{WP}+$ highL $+\mathrm{BAO}$ results [30].

\section{Statefinder Diagnostic}

The dark energy properties are model-dependent. In order to distinguish between different types of dark energy models, in 2003 Sahni et al. [33] have suggested a diagnostic proposal by introducing a new pair of parameters $\{r, s\}$, called statefinder parameters. These parameters can be constructed by using both the second and third derivatives of the expansion factor. 


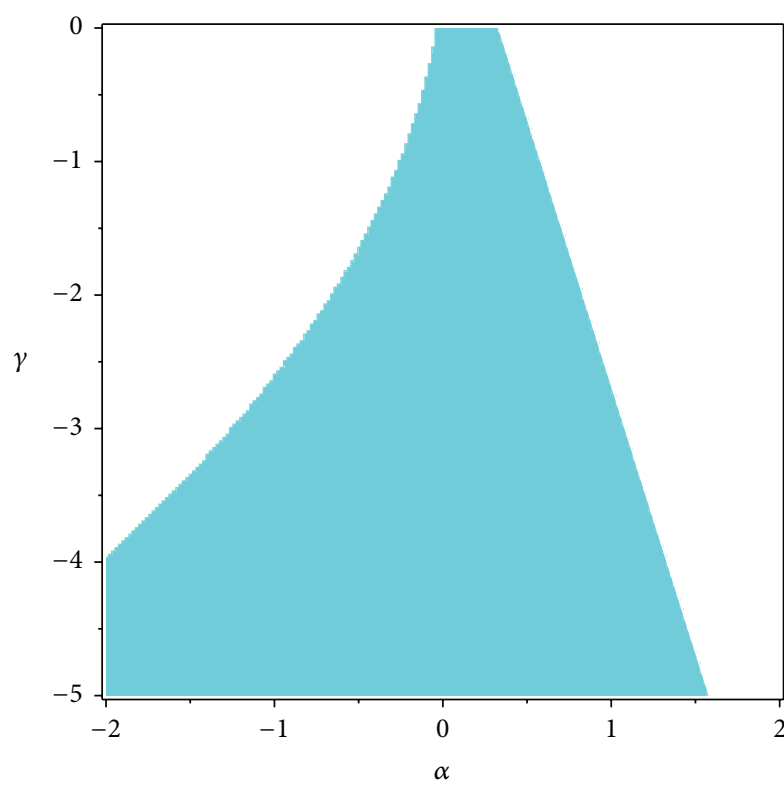

(a)

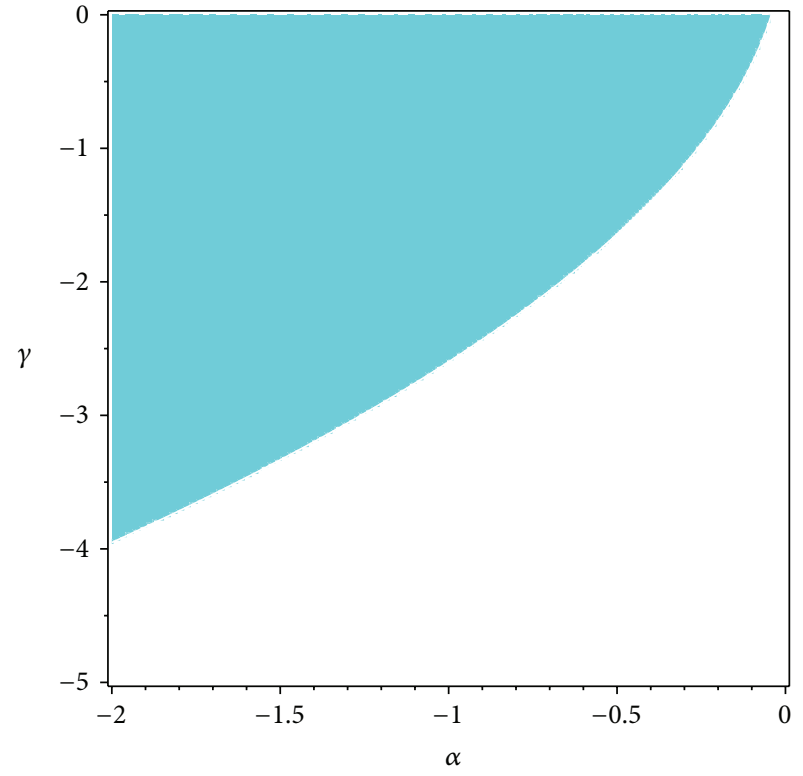

(b)

Figure 5: In the $\gamma-\alpha$ space with $\xi=1 / 6$, the critical points $C_{ \pm}$are stable nodes for the shaded region (a). Also the critical points $C_{ \pm}$with $\xi=1 / 6$ are stable spirals for shaded region (b).

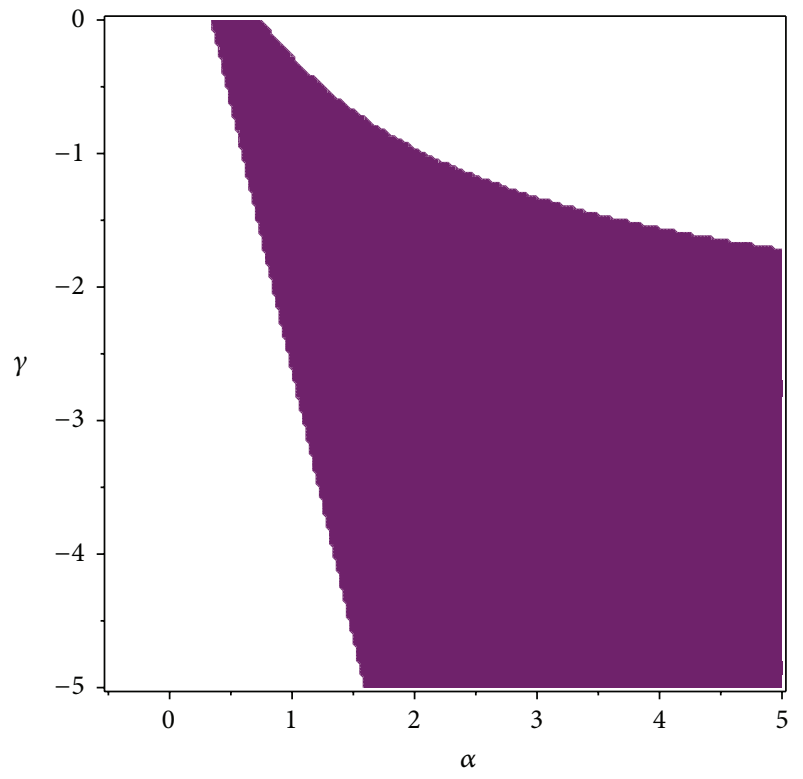

(a)

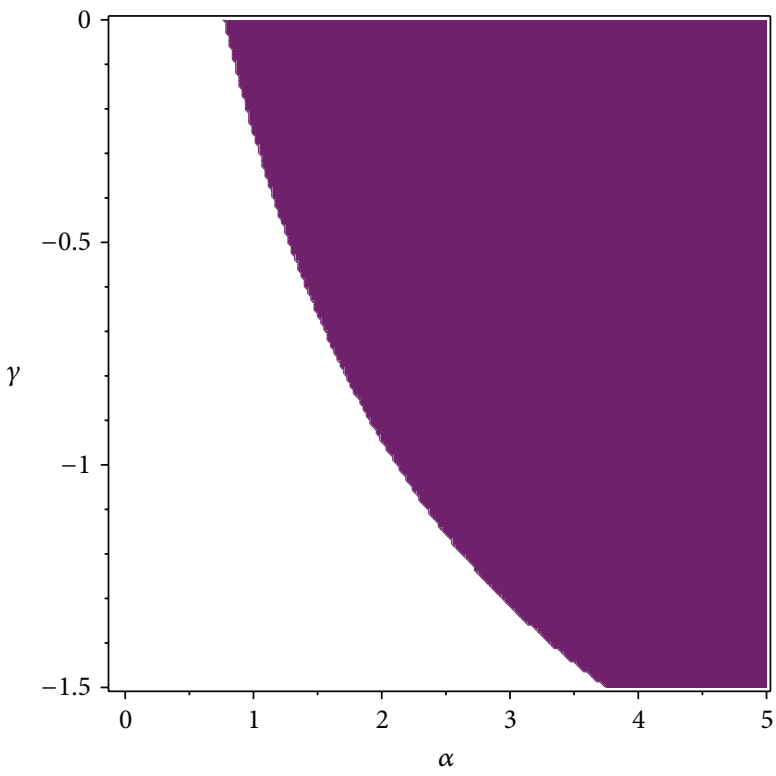

(b)

Figure 6: In the $\gamma-\alpha$ space with $\xi=1 / 6$, the critical points $D_{ \pm}$are stable nodes for the shaded region (a). Also the critical points $D_{ \pm}$with $\xi=1 / 6$ are stable spirals for shaded region (b).

The second derivative gives the deceleration parameter which has the following form in a spatially flat universe:

$$
q=-\frac{\ddot{a}}{a H^{2}}=-\left(1+\frac{\dot{H}}{H^{2}}\right)
$$

The statefinder pair, $\{r, s\}$, are defined as

$$
\begin{gathered}
r=\frac{\dddot{a}}{a H^{3}}=\frac{\ddot{H}}{H^{3}}-3 q-2, \\
s=\frac{r-1}{3(q-(1 / 2))} .
\end{gathered}
$$




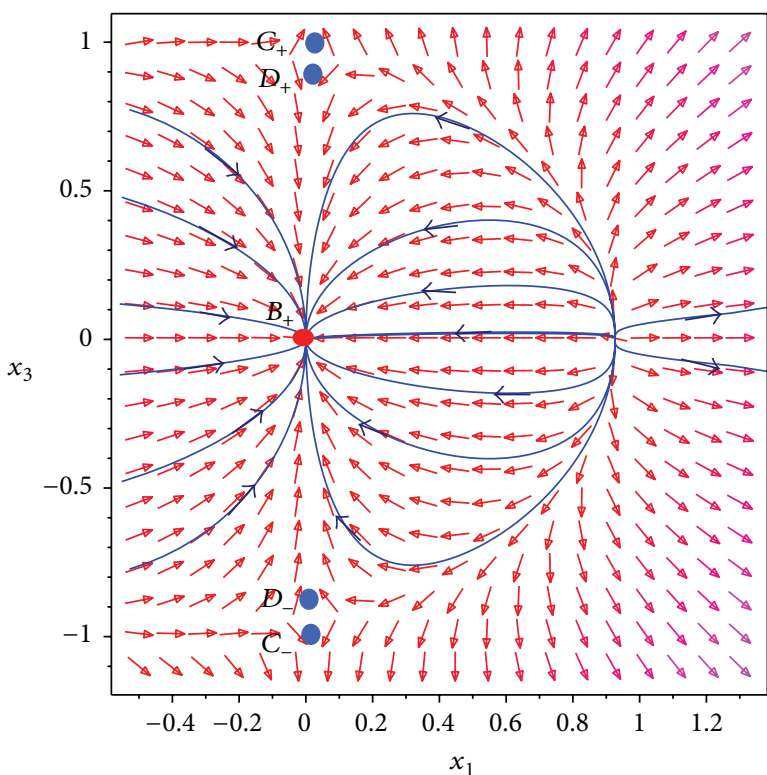

(a)

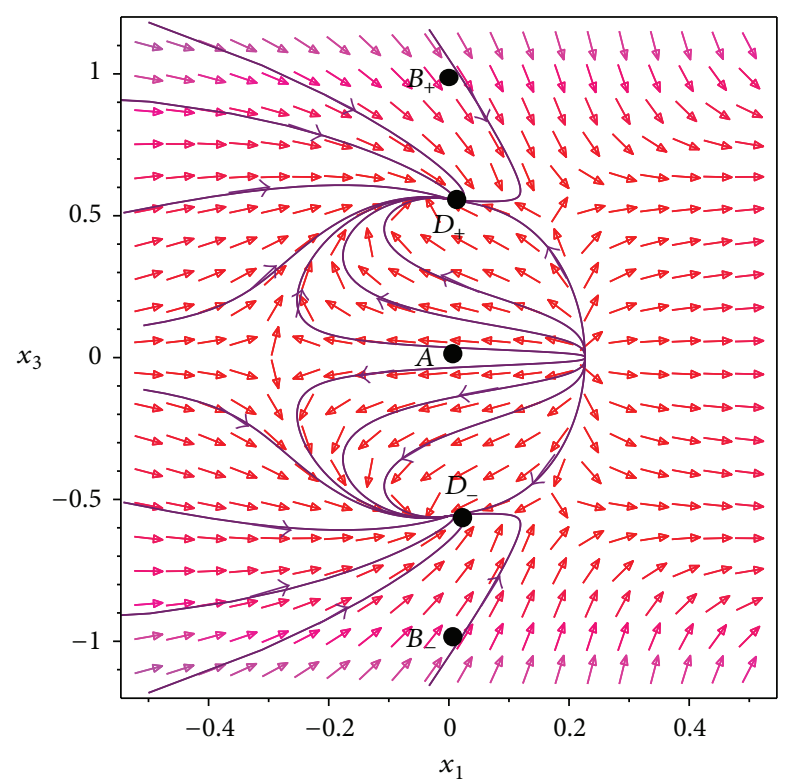

(b)

Figure 7: (a) The phase plane for the critical $B_{+}$with $\gamma=1, \lambda=0.5, \alpha=0.1$, and $\xi=1 / 6$. The critical point $B_{+}(0,0)$ is a stable node (a sink) so that the quintessence dominated solution is the future attractor. The critical points $c_{ \pm}(0, \pm 1)$ and $D_{ \pm}(0, \pm 0.9)$ are saddle points. (b) The phase plane for the critical points $D_{+}$with $\gamma=-0.1, \lambda=1, \alpha=1.15$, and $\xi=1 / 6$. The critical points $D_{+}(0, \pm 0.55)$ are stable nodes (sinks) so that the quintessence dominated solution is the late time attractor. The critical points $A(0,0)$ and $B_{ \pm}(0, \pm 1)$ are saddle points.

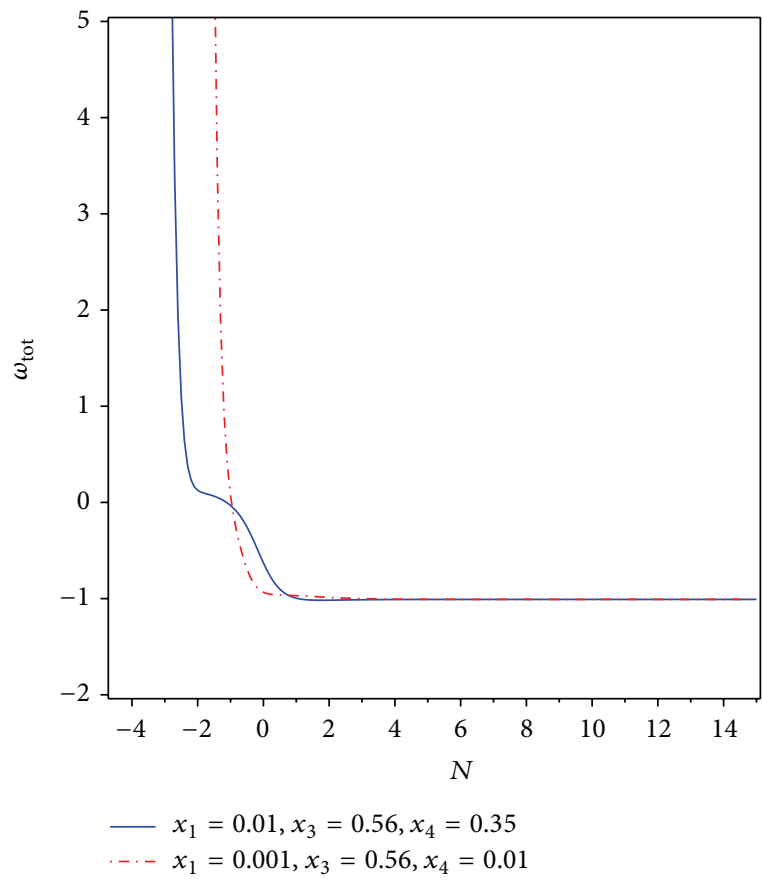

(a)

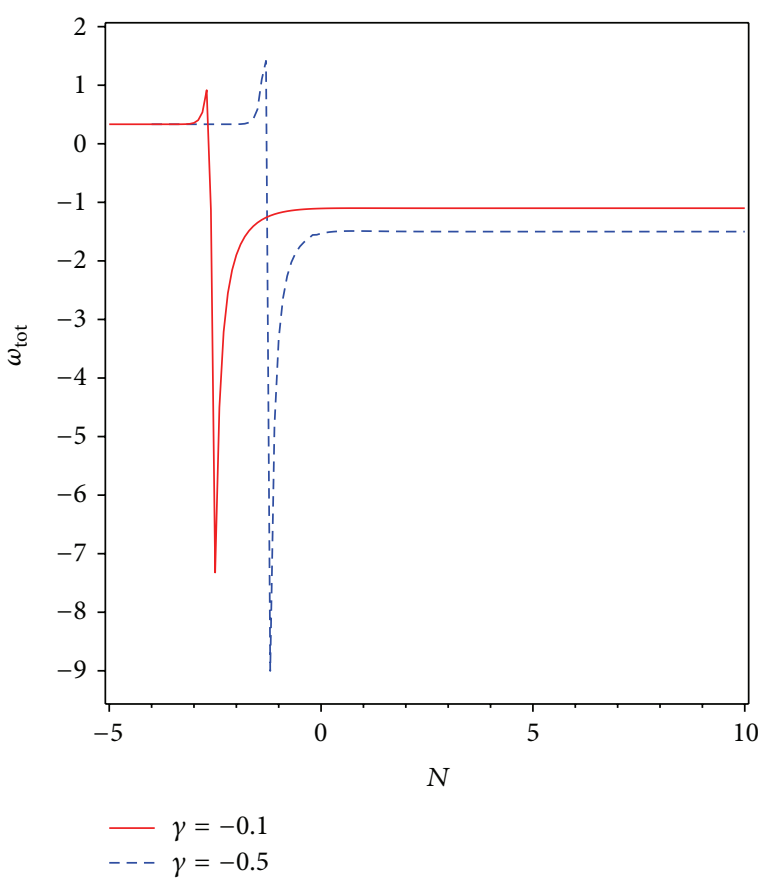

(b)

Figure 8: Effective equation of state parameter versus the cosmic time for the critical point $B_{+}$with $\gamma=1, \lambda=0.5, \alpha=0.1$ and $\xi=1 / 6$ and initial values as given in figure (a). (b) is for the effective equation of state parameter for the critical points $D_{ \pm}$with $\gamma=-0.1, \lambda=1, \alpha=1.15$ and $\xi=1 / 6$ and $x_{1}=0, x_{3}=0.56, x_{4}=0.83$ as initial values. 
The statefinder diagnostic tool depends on the scale factor. This shows that the statefinder parameters are geometrical in essence. Nowadays, the various dark energy models have been considered from the statefinder diagnostic point of view. The different dark energy models have various evolutionary trajectories in $\{s, r\}$ plane. So, the statefinder tool can distinguish between alternative models. Furthermore, by using the statefinder parameters, we can study the expansion history of the universe by using higher derivatives of the scale factor. The $\Lambda C D M$ scenario corresponds to a critical point in the $r$-s phase diagram with $\{r, s\}_{\Lambda C D M}=\{1,0\}$. By drawing the trajectories in the $r$-s phase plane, the discrepancy of the model from $\Lambda C D M$ can be investigated [34]. We can rewrite (41) as

$$
r=\frac{d}{d N}\left[\frac{\dot{H}}{H^{2}}\right]+2\left[\frac{\dot{H}}{H^{2}}\right]^{2}+3\left[\frac{\dot{H}}{H^{2}}\right]+1 .
$$

In a minimally coupled quintessence field with the dark matter, by substituting (11) into (43), $r$ is obtained to be as follows

$$
r=-3 \gamma x_{3} x_{3}^{\prime}-6 x_{1} x_{1}^{\prime}+2\left[\frac{\dot{H}}{H^{2}}\right]^{2}+3 \frac{\dot{H}}{H^{2}}+1,
$$

where a prime denotes derivative with respect to $N=\ln a(t)$.

Also $s$ parameter can be derived by substituting (44), (40), and (11) into (42). Due to its lengthy form, we avoid presenting this quantity explicitly. Now, we examine the numerical analysis of the statefinder diagnostic. Figure 9 for the late time stable scaling solutions $E_{ \pm}$, shows the trajectories of $\{r, s\},\{q, s\}$, and $\{q, r\}$ phase plane for two different $\gamma$ and $\lambda$ parameters. If $\gamma=0.5$ and $\lambda=1.48$, the initial values will be $x_{01}=0.41$ and $x_{03}=0.56$ with $\left(x_{03}^{2}=\Omega_{m}=0.315\right)$ for blue, dashed line. For $\gamma=0.05$ and $\lambda=0.47$, the initial values will be $x_{01}=0.13$ and $x_{03}=0.56$ for purple solid line and this is in agreement with Planck data [30]. As the figure illustrates with various $\gamma$ and $\lambda$ parameters, the trajectories evolve differently. One can choose small values of $\gamma$ and $\lambda$ in order to approach the $\Lambda C D M$ model in this framework. We note also that in this platform one can choose the suitable values of $\gamma$ and $\lambda$ in order to find more viable values of $\Omega_{m}$ in comparison with recent observations.

After studying statefinder diagnostic for the case with minimal coupling between the quintessence field and dark matter, now we extend our analysis to the nonminimal coupling between the dark energy component and the scalar curvature. The $r$ parameter can be obtained by replacing (24) into (43)

$$
\begin{aligned}
r=1 & \\
& +\frac{-3 \gamma x_{3} x_{3}^{\prime}-6 x_{1} x_{1}^{\prime}-4 \sqrt{6 \xi}\left(x_{4} x_{1}^{\prime}+x_{1} x_{4}^{\prime}\right)+12 \xi x_{1} x_{1}^{\prime}}{1-x_{4}^{2}+6 \xi x_{4}^{2}} \\
& +\left(6 \lambda \sqrt{\xi} x_{4}\left[\left(x_{1}^{2}+x_{3}^{2}+x_{4}^{2}+2 \sqrt{6 \xi} x_{1} x_{4}-1\right)^{1 / 2}\right]\right. \\
& \left.\quad \times\left[x_{1} x_{1}^{\prime}+x_{3} x_{3}^{\prime}+x_{4} x_{4}^{\prime}+\sqrt{6 \xi}\left(x_{1}^{\prime} x_{4}+x_{1} x_{4}^{\prime}\right)\right]\right)
\end{aligned}
$$

$$
\begin{aligned}
& \times\left(1-x_{4}^{2}+6 \xi x_{4}^{2}\right)^{-1} \\
& +\frac{3 \lambda \sqrt{\xi}\left(1-x_{1}^{2}-x_{3}^{2}-x_{4}^{2}-2 \sqrt{6 \xi} x_{1} x_{4}\right) x_{4}^{\prime}-24 \xi x_{3} x_{4}^{\prime}}{1-x_{4}^{2}+6 \xi x_{4}^{2}} \\
& +\left[\left(\frac{2 x_{4} x_{4}^{\prime}-12 \xi x_{4} x_{4}^{\prime}}{1-x_{4}^{2}+6 \xi x_{4}^{2}}\right)+3\right] \frac{\dot{H}}{H^{2}}+2\left(\frac{\dot{H}}{H^{2}}\right)^{2} .
\end{aligned}
$$

As we have mentioned previously, $s$ parameter can be obtained by replacing (45), (40), and (24) in (42). Now we present the numerical analysis of the statefinder diagnostic. Figure 10 for the stable node $B_{+}$shows the trajectories of $\{r, s\},\{q, s\}$, and $\{q, r\}$ phase planes for two different initial values with $\gamma=1, \lambda=1$, and $\xi=1 / 6$. As this figure indicates, the model with ordinary dark mater $(\gamma=1)$ and a nonminimally coupled quintessence field, the trajectories of $\{r, s\},\{q, s\}$, and $\{q, r\}$ in phase plane reach a stable point in the future, that is, corresponding to the $\triangle C D M$ model $(\{r, s, q\}=\{1,0,-1\})$.

Now we study statefinder diagnostic for a general model with dark energy nonminimally coupled to both dark matter and scalar curvature. Similar to our previous treatment, the $r$ parameter can be derived by replacing (37) into (43)

$$
\begin{aligned}
r=1+( & -3 \gamma x_{3} x_{3}^{\prime}-6 x_{1} x_{1}^{\prime}-4 \sqrt{6 \xi}\left(x_{4} x_{1}^{\prime}+x_{1} x_{4}^{\prime}\right) \\
& \left.+12 \xi x_{1} x_{1}^{\prime}+6 \alpha\left(2 x_{4} x_{4}^{\prime} x_{3}^{2}+2 x_{4}^{2} x_{3} x_{3}^{\prime}\right)\right) \\
& \times\left(1-x_{4}^{2}+6 \xi x_{4}^{2}\right)^{-1} \\
+ & \left(6 \lambda \sqrt{\xi} x_{4}\left[\left(x_{1}^{2}+x_{3}^{2}+x_{4}^{2}+2 \sqrt{6 \xi} x_{1} x_{4}-1\right)^{1 / 2}\right]\right. \\
& \left.\left.\times\left(1-x_{4}^{2}+6 \xi x_{4}^{2}\right)^{-1} x_{1}^{\prime}+x_{3} x_{3}^{\prime}+x_{4} x_{4}^{\prime}+\sqrt{6 \xi}\left(x_{1}^{\prime} x_{4}+x_{1} x_{4}^{\prime}\right)\right]\right) \\
+ & \frac{3 \lambda \sqrt{\xi}\left(1-x_{1}^{2}-x_{3}^{2}-x_{4}^{2}-2 \sqrt{6 \xi} x_{1} x_{4}\right) x_{4}^{\prime}-24 \xi x_{3} x_{4}^{\prime}}{1-x_{4}^{2}+6 \xi x_{4}^{2}} \\
+ & {\left[\left(\frac{2 x_{4} x_{4}^{\prime}-12 \xi x_{4} x_{4}^{\prime}}{1-x_{4}^{2}+6 \xi x_{4}^{2}}\right)+3\right] \frac{\dot{H}}{H^{2}}+2\left(\frac{\dot{H}}{H^{2}}\right)^{2} . }
\end{aligned}
$$

The parameter $s$ can be calculated by replacing (46), (40), and (37) into (42). For this model we analyze numerically the statefinder diagnostic for critical points $B_{+}$, and $D_{ \pm}$. If we consider $0<\gamma<2$, then the critical points $D_{ \pm}$for the late time scaling solution will be saddle points (see Table 5). In addition, by considering the attractor points $B_{ \pm}$, the model reaches the $\Lambda C D M$ model in the future. For stable node $B_{+}$, the trajectories of $\{r, s\},\{q, s\}$, and $\{q, r\}$ in phase plane with two initial conditions are drawn in Figure 11. In these figures we considered two 


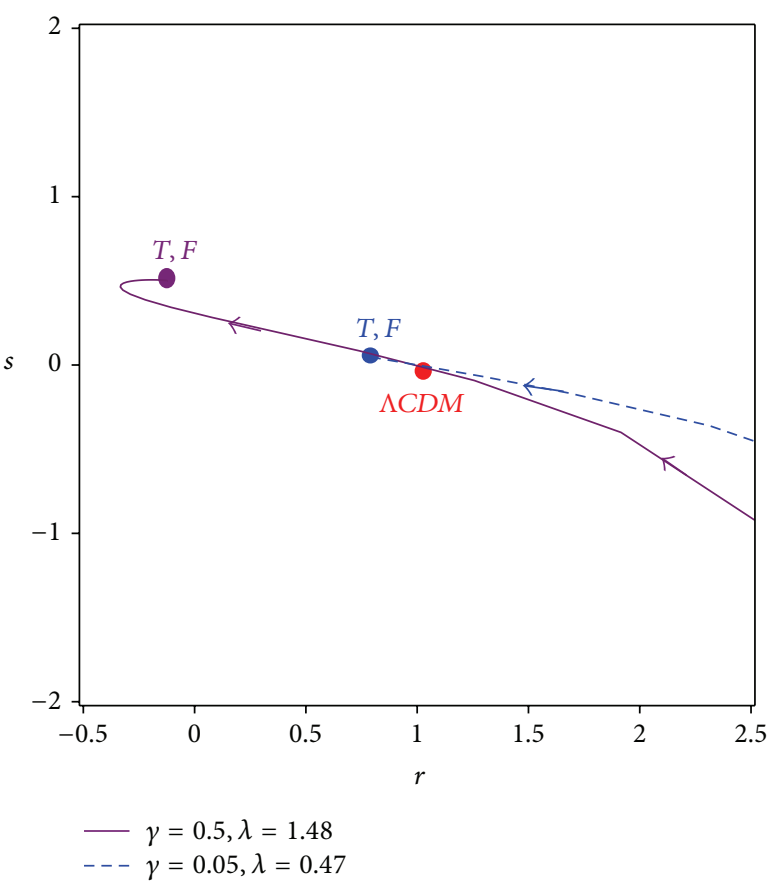

(a)

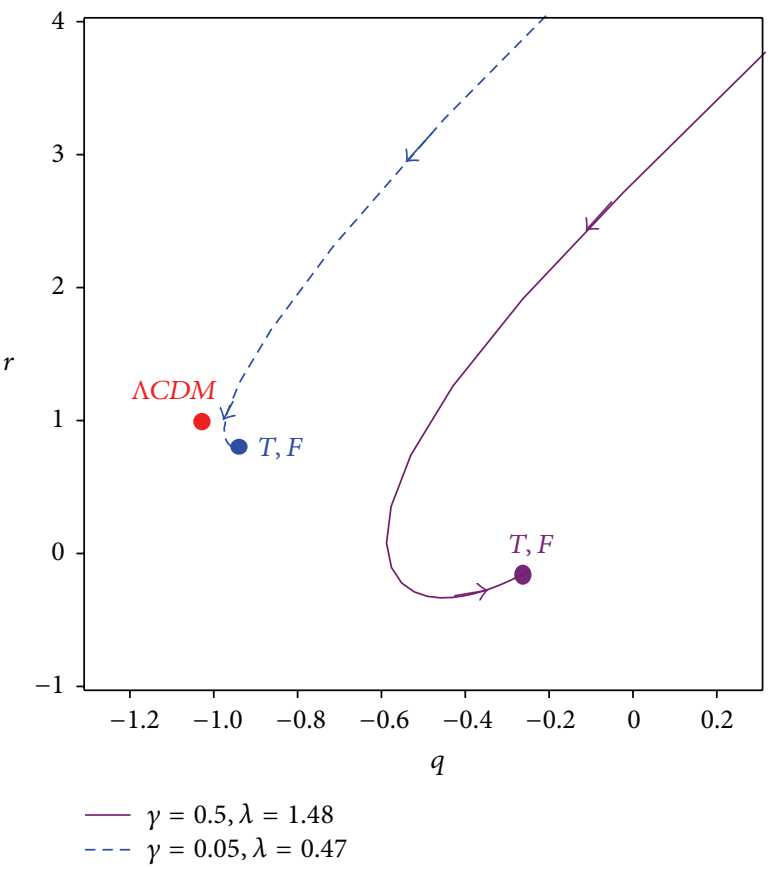

(b)

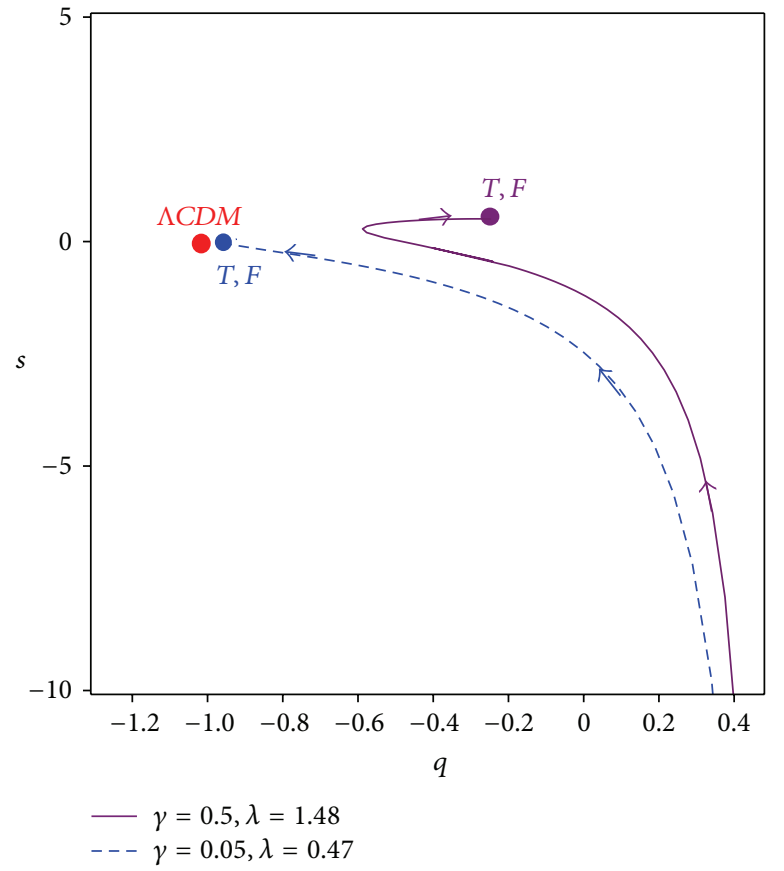

(c)

FIGURE 9: Trajectories of the stable nodes $E_{ \pm}$in $\{r, s\}$ phase plane with initial values $x_{1}=0.41$ and $x_{3}=0.56$ for the blue dashed line, and $x_{1}=0.13$ and $x_{3}=0.56$ for purple solid line with the specified values of $\gamma$ and $\lambda$ (a). Point $T$ shows the late time values of $\{r, s\}$ in this model. Point $F$ is the stable state of $\{r, s\}$ in the future. The value of the statefinder $\{r, s\}$ in the $\Lambda C D M$ scenario is shown by $\Lambda C D M$ point. (b) shows the trajectories of the stable nodes $E_{ \pm}$in $\{q, r\}$ phase plane with the same initial values as for (a). (c) is devoted to $\{q, s\}$ phase plane.

different sets of initial values for $x_{1}, x_{3}$, and $x_{4}$ as have been shown in figures and we have set also $\xi=1 / 6$, $\lambda=0.5, \alpha=0.1$, and $\gamma=1$ (for ordinary dark matter). These two different initial values lead to the trajectories that evolve to the same fixed point in the future. For stable nodes
$D_{ \pm}$, with $\gamma<0$ (corresponding to nonordinary dark matter) we have late time scaling solutions. The trajectories of $\{r, s\}$, $\{q, s\}$, and $\{q, r\}$ in phase plane with two different values of the $\gamma$ parameter, but with the same initial conditions, $x_{1}=0, x_{3}=$ 0.56 , and $x_{4}=0.83$, have been shown in Figure 12. As this 


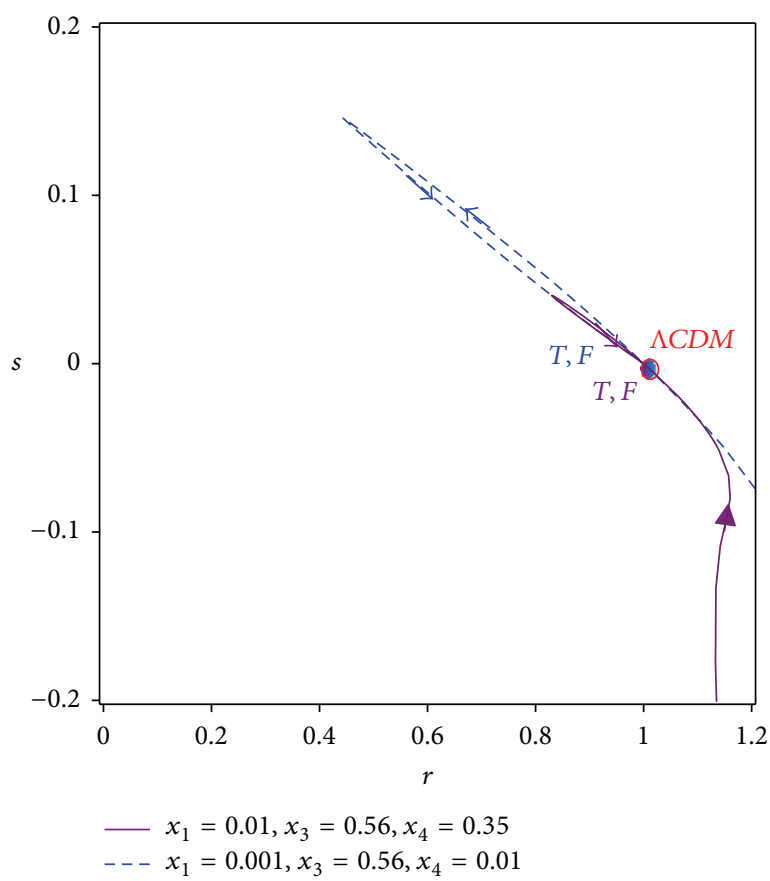

(a)

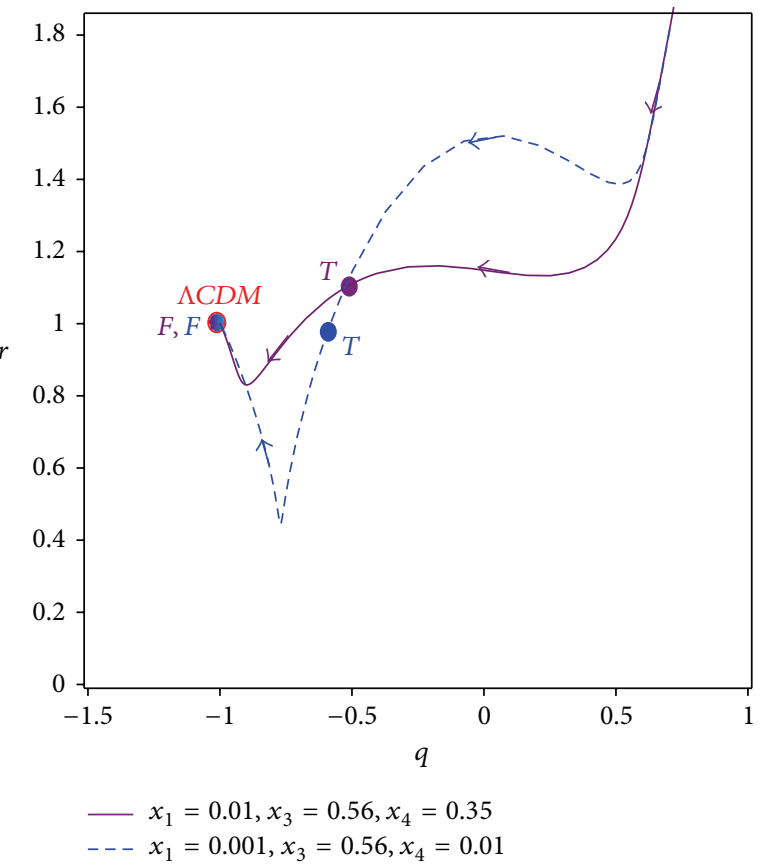

(b)

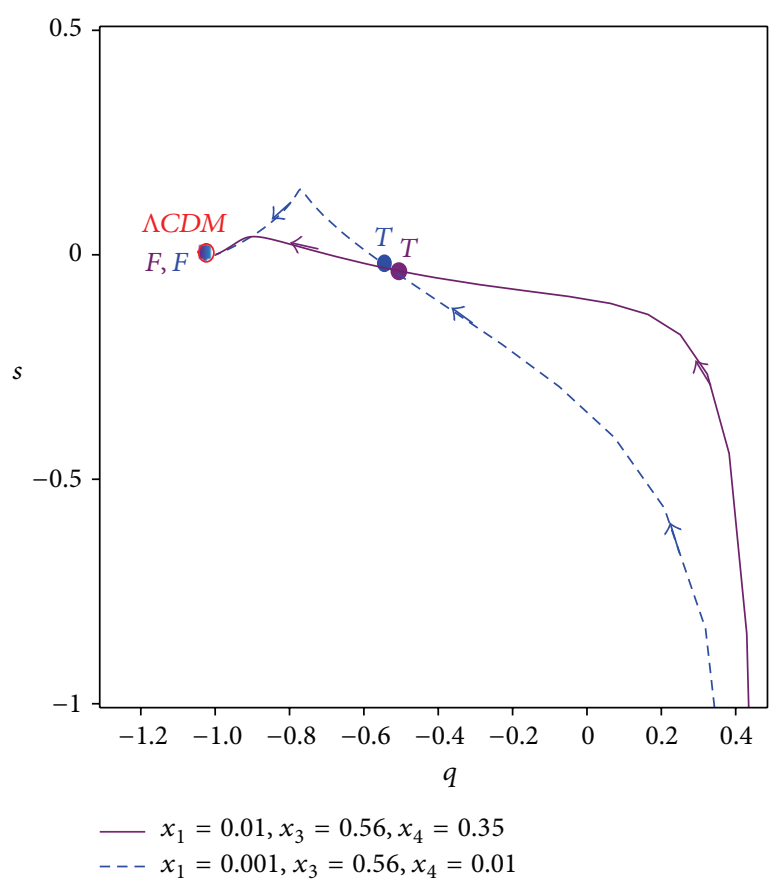

(c)

Figure 10: Trajectories in $\{r, s\},\{q, r\}$, and $\{q, s\}$ phase planes for the stable node $B_{+}$with $\lambda=1$ and $\gamma=1$ (corresponding to ordinary dark matter) with two sets of initial conditions.

figure illustrates, with different values of the $\gamma$ parameter the trajectories evolve differently. With both adopted values of the $\gamma$ parameter, the model approaches the $\Lambda C D M$ model in the late time and also the future. However, it is possible to choose the values of $\gamma$ so that the model tends to the $\Lambda C D M$ model at late time or future.

\section{Summary and Conclusion}

In this paper we have studied an interacting model in which the dark sectors (dark matter and dark energy) are coupled to each other in a background gravitational field which is nonminimally coupled to the dark energy. We have shown 


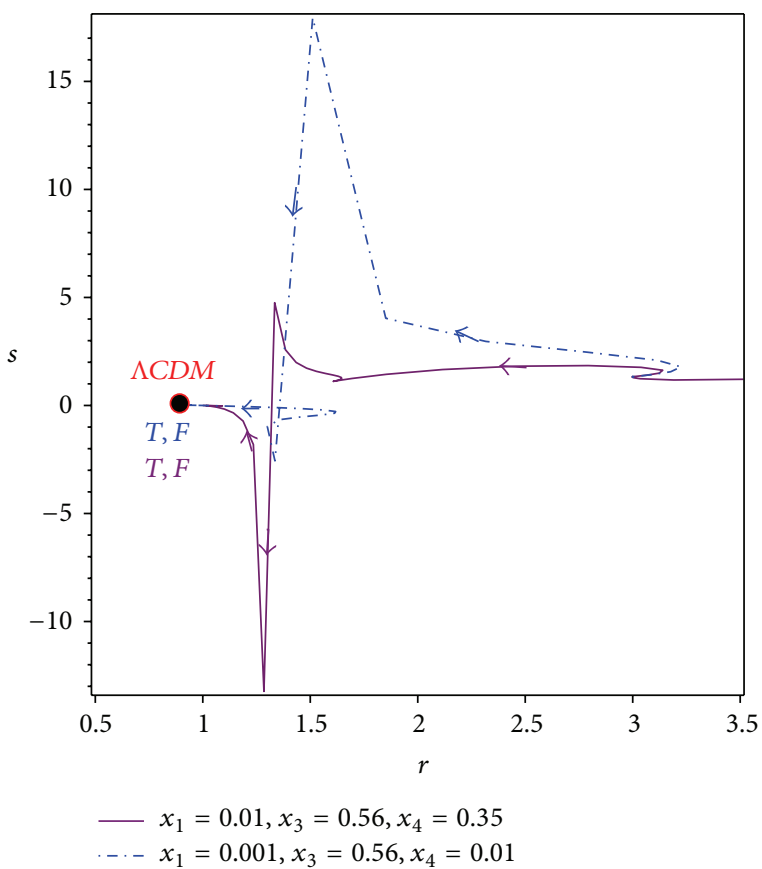

(a)

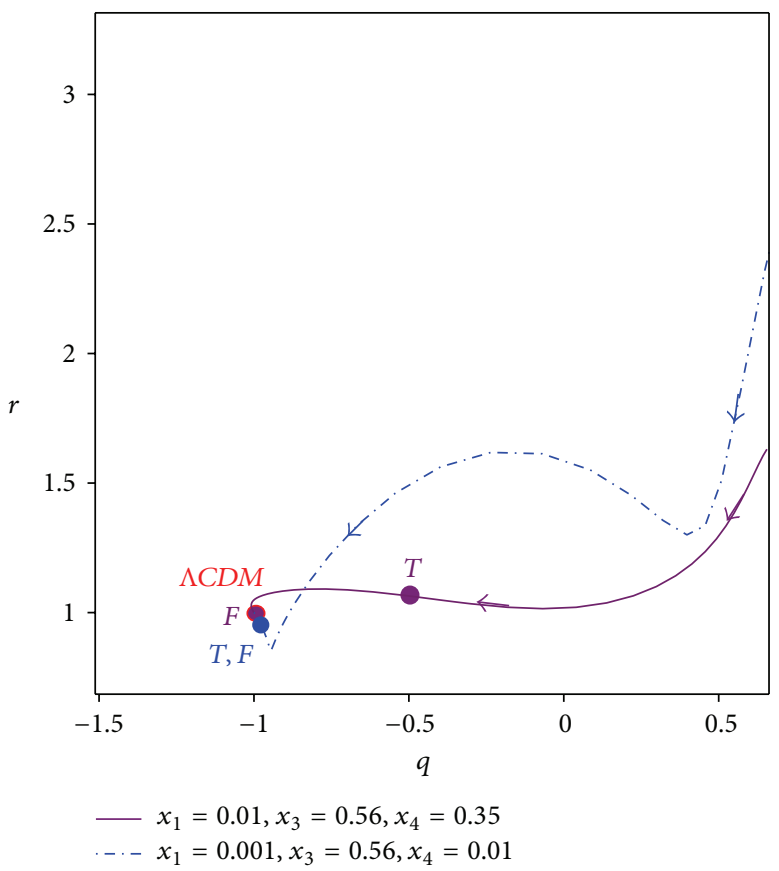

(b)

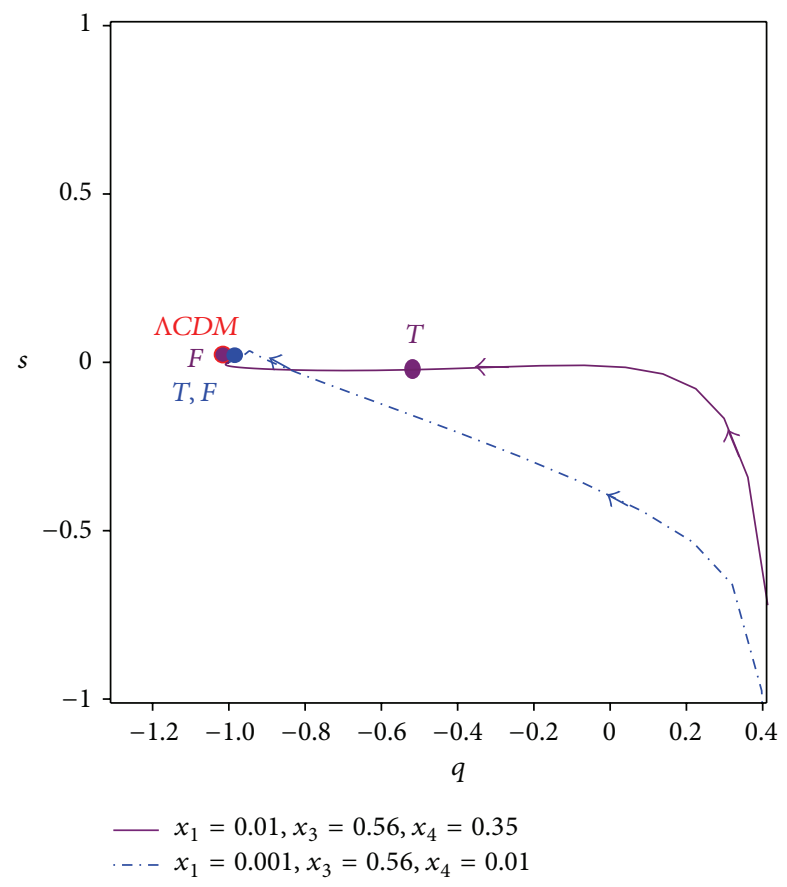

(c)

Figure 11: Trajectories in $\{r, s\},\{q, r\}$, and $\{q, s\}$ phase planes for the stable node $B_{+}$with $\xi=1 / 6, \lambda=0.5, \alpha=0.1$, and $\gamma=1$ with two sets of initial conditions as specified in the figures.

the possibility of having scaling solutions in this setup. These solutions are capable of addressing the coincidence problem in a manner much similar to the chameleon mechanism. In fact, we have introduced the idea that $\gamma$, as the barotropic index, is capable of getting negative values for nonordinary dark matter (or in some nonstandard situations). This assumption opens new windows in the issue of dark energy interacting with dark matter. With this assumption, it is possible to have complex sound velocity in the medium much similar to the complex index of refraction in optical media.

In summary, in the first part of the paper we have considered a minimal model with no interaction between 


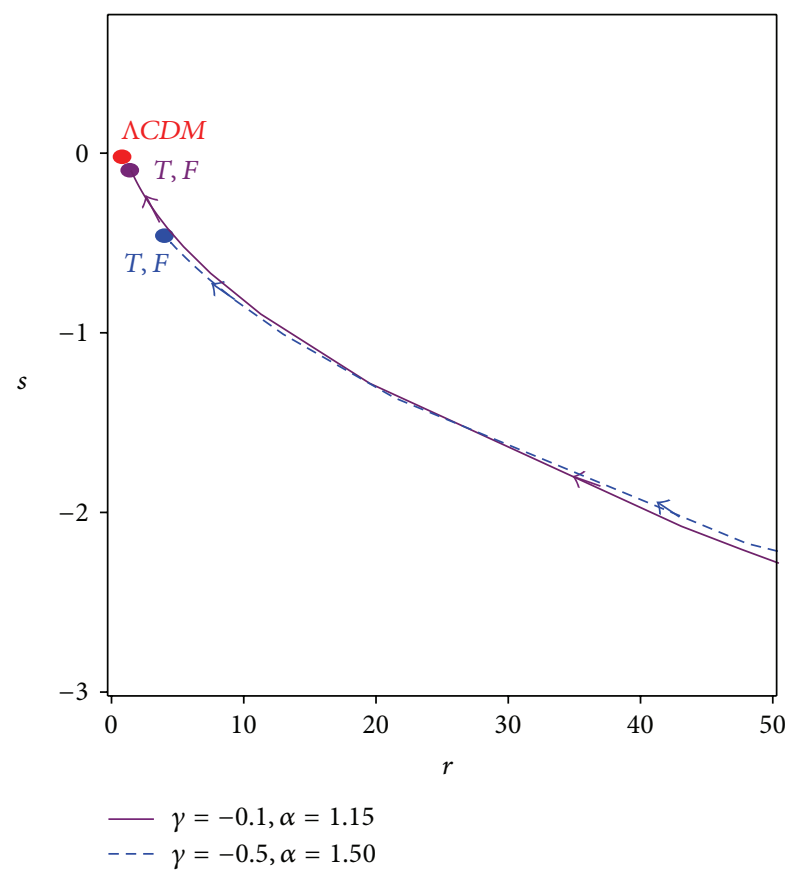

(a)

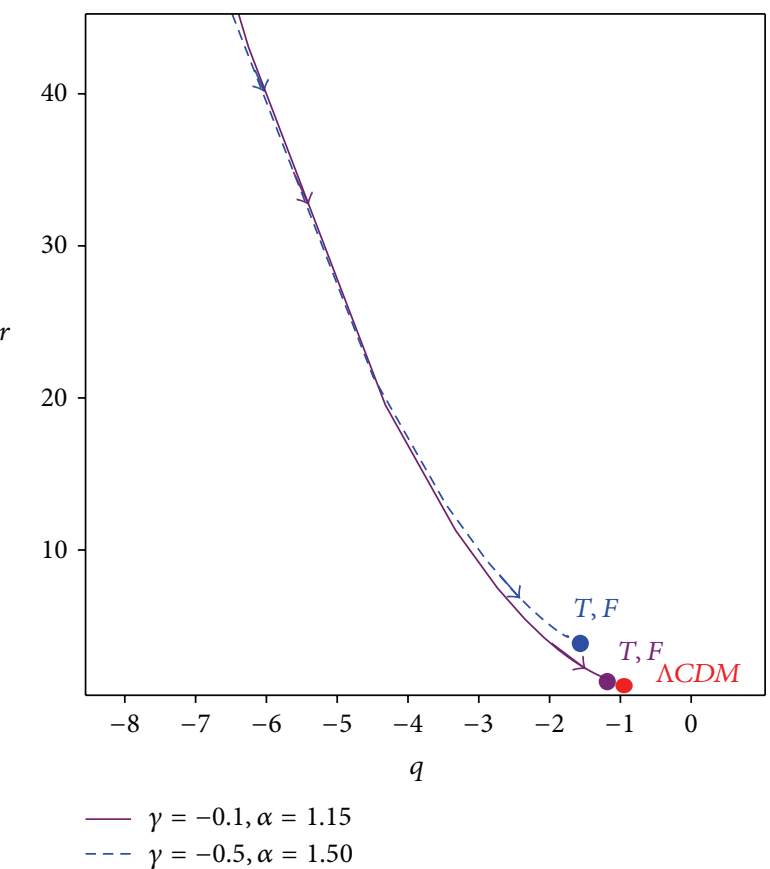

(b)

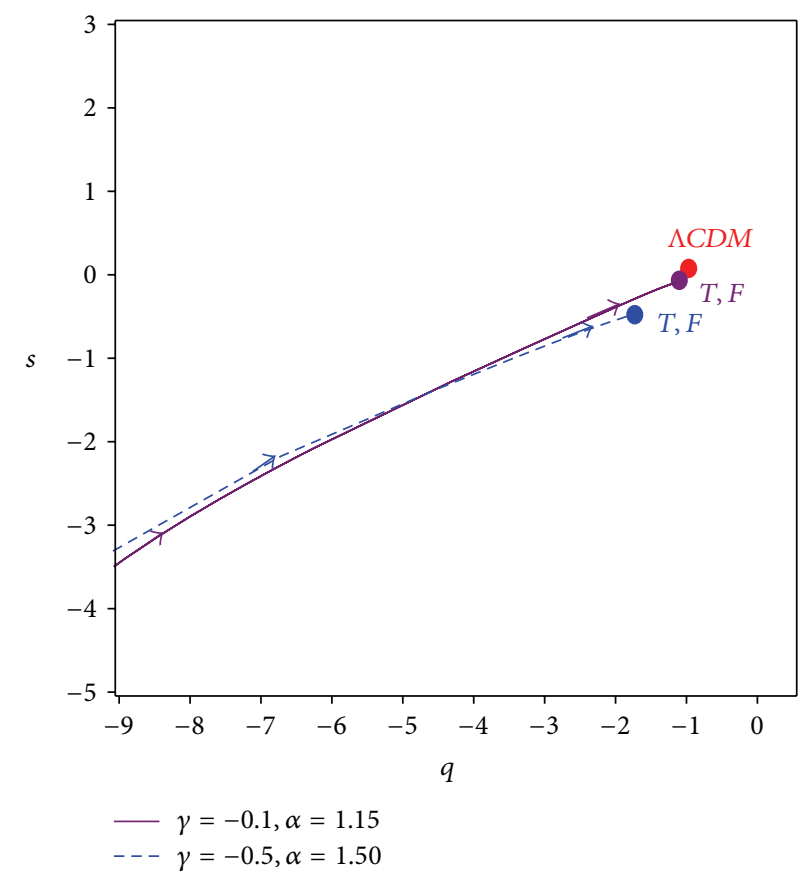

(c)

FIgURE 12: Trajectories in $\{r, s\},\{q, r\}$, and $\{q, s\}$ phase planes for the stable nodes $D_{ \pm}$with $\xi=1 / 6, \lambda=1$ and initial conditions $x_{1}=0$, $x_{3}=0.56$, and $x_{4}=0.83$.

the dark sectors. In this case there is no crossing of the phantom divide. In the next step we considered nonminimal coupling between dark energy and gravitational sector but without any interaction between the dark sectors. In this case, there is no attractor solution for late time. There is also no possibility for transition to phantom phase. In the last step we supposed a general model with interaction between the dark sectors and also between dark energy and scalar curvature. This general model in the spirit of scalar-tensor theories provides a lot of interesting facilities: there is a possiblity to cross the phantom divide line by the equation of state parameter in some subspaces of the model parameter space, it is possible to address the coincidence problem in a natural manner and finally there is a late time attractor which goes 
to $\Lambda C D M$ in the future via statefinder diagnostic analysis. In each step we have compared our results with observational data from Planck + WP + highL + BAO joint data set.

\section{Appendix}

The values of the parameters $G_{i}$ introduced in Tables 4 and 6 are as follows:

$$
\begin{aligned}
& G_{1,2}= \pm \frac{1}{2}\left(\mp 27 \xi \lambda^{2}\right. \\
& +\left(-3\left(9 \xi \lambda^{2}+24 \xi-4\right)\right. \\
& \times\left(96 \xi^{3 / 2} \sqrt{\lambda^{2}+4 \xi}+192 \xi^{2}+21 \xi \lambda^{2}\right. \\
& \left.\left.-32 \sqrt{\xi\left(\lambda^{2}+4 \xi\right)-72 \xi+12}\right)\right)^{1 / 2} \\
& \mp 72 \xi \pm 12) \\
& \times\left(9 \xi \lambda^{2}+24 \xi-4\right)^{-1} \\
& G_{3,4}= \pm \frac{1}{2}\left(\mp 27 \xi \lambda^{2}\right. \\
& +\left(3\left(9 \xi \lambda^{2}+24 \xi-4\right)\right. \\
& \times\left(96 \xi^{3 / 2} \sqrt{\lambda^{2}+4 \xi}+192 \xi^{2}+21 \xi \lambda^{2}\right. \\
& \left.\left.-32 \sqrt{\xi\left(\lambda^{2}+4 \xi\right)-72 \xi+12}\right)\right)^{1 / 2} \\
& \mp 72 \xi \pm 12) \\
& \times\left(9 \xi \lambda^{2}+24 \xi-4\right)^{-1} \\
& G_{5,6}=\left( \pm 2916\left(\left(\xi-\frac{1}{6}\right) \gamma+\frac{2}{0}-\frac{4}{3} \xi+\frac{2}{3} \alpha\right)\right. \\
& \times\left(\left(\xi-\frac{1}{6}\right) \gamma^{3}+\left(\frac{2}{3} \alpha+\frac{8}{9}\right) \gamma^{2}\right. \\
& +\left(\frac{8}{9} \alpha-\frac{14}{9}-\frac{44}{9} \xi\right) \gamma-\frac{56}{27} \alpha \\
& \left.\left.+\frac{8}{9}+\frac{112}{27} \xi\right)\right)^{1 / 2} \\
& \times((72 \xi-12) \gamma-96 \xi+48 \alpha+16)^{-1} \\
& +\left((54 \xi-9) \gamma^{2}+(30-180 \xi+36 \alpha) \gamma\right. \\
& +144 \xi-72 \alpha-24) \\
& \times((72 \xi-12) \gamma-96 \xi+48 \alpha+16)^{-1}
\end{aligned}
$$

\section{Conflict of Interests}

The authors declare that there is no conflict of interests regarding the publication of this paper.

\section{References}

[1] A. G. Riess, A. V. Filippenko, P. Challis et al., "Observational evidence from supernovae for an accelerating universe and a cosmological constant," The Astronomical Journal, vol. 116, no. 3, p. 1009, 1998.

[2] S. Perlmutter, G. Aldering, G. Goldhaber et al., "Measurements of $\Omega$ and $\Lambda$ from 42 High-Redshift Supernovae," The Astrophysical Journal, vol. 517, no. 2, pp. 565-586, 1999.

[3] A. G. Riess, L.-G. Strolger, J. Tonry et al., "Type Ia Supernova discoveries at $z>1$ from the hubble space telescope: evidence for past deceleration and constraints on dark energy evolution," The Astrophysical Journal, vol. 607, no. 2, pp. 665-687, 2004.

[4] P. Astier, J. Guy, N. Regnault et al., "The supernova legacy survey: measurement of $\Omega_{M}, \Omega_{\Lambda}$ and $w$ from the first year data set," Astronomy \& Astrophysics, vol. 447, no. 1, pp. 31-48, 2006.

[5] D. N. Spergel, R. Bean, O. Doré et al., "Three-year Wilkinson microwave anisotropy probe (WMAP) observations: implications for cosmology," The Astrophysical Journal, vol. 170, no. 2, p. 377, 2007.

[6] E. Komatsu, J. Dunkley, M. R. Nolta et al., "Five-year Wilkinson Microwave Anisotropy Probe (WMAP) observations: cosmological interpretation," The Astrophysical Journal Supplement Series, vol. 180, no. 2, p. 330, 2009.

[7] T. P. Sotiriou and V. Faraoni, " $f(R)$ theories of gravity," Reviews of Modern Physics, vol. 82, no. 1, pp. 451-497, 2010.

[8] S. Nojiri and S. D. Odintsov, "Unified cosmic history in modified gravity: from $F(R)$ theory to Lorentz non-invariant models," Physics Reports, vol. 505, no. 2-4, pp. 59-144, 2011.

[9] T. Padmanabhan, "Cosmological constant-the weight of the vacuum," Physics Reports, vol. 380, no. 5-6, pp. 235-320, 2003.

[10] S. M. Carroll, "The cosmological constant," Living Reviews in Relativity, vol. 4, p. 1, 2001.

[11] V. Sahni and A. Starobinsky, "The case for a positive cosmological $\lambda$-term," International Journal of Modern Physics D, vol. 9, no. 4, p. 373, 2000.

[12] P. J. Peebles and B. Ratra, "The cosmological constant and dark energy," Reviews of Modern Physics, vol. 75, no. 2, pp. 559-606, 2003.

[13] R. R. Caldwell, R. Dave, and P. J. Steinhardt, "Cosmological imprint of an energy component with general equation of state," Physical Review Letters, vol. 80, no. 8, pp. 1582-1585, 1998.

[14] I. Zlatev, L. Wang, and P. J. Steinhardt, "Quintessence, cosmic coincidence, and the cosmological constant," Physical Review Letters, vol. 82, no. 5, pp. 896-899, 1999.

[15] R. R. Caldwell, M. Kamionkowski, and N. N. Weinberg, "Phantom energy: dark energy with $w<-1$ causes a cosmic doomsday," Physical Review Letters, vol. 91, Article ID 071301, 2003.

[16] A. Sen, "Tachyon matter," Journal of High Energy Physics, no. 7, article 65, 2002.

[17] A. Sen, "Field theory of tachyon matter," Modern Physics Letters A: Particles and Fields, Gravitation, Cosmology, Nuclear Physics, vol. 17, no. 27, pp. 1797-1804, 2002.

[18] E. J. Copeland, M. Sami, and S. Tsujikawa, "Dynamics of dark energy," International Journal of Modern Physics D, vol. 15, no. 11, pp. 1753-1935, 2006. 
[19] T. Gonzalez, G. Leon, and I. Quiros, "Dynamics of quintessence models of dark energy with exponential coupling to dark matter," Classical and Quantum Gravity, vol. 23, no. 9, pp. 31653179, 2006.

[20] L. Amendola and D. Tochini, "Baryon bias and structure formation in an accelerating universe," Physical Review D, vol. 66, Article ID 043528, 2002.

[21] L. Amendola, "Coupled quintessence," Physical Review D, vol. 62, no. 4, Article ID 043511, 10 pages, 2000.

[22] W. Zimdahl, D. Pavón, and L. P. Chimento, "Interacting quintessence," Physics Letters B, vol. 521, no. 3-4, pp. 133-138, 2001.

[23] L. P. Chimento, A. S. Jakubi, D. Pavón, and W. Zimdahl, "Interacting quintessence solution to the coincidence problem," Physical Review D, vol. 67, Article ID 083513, 2003.

[24] L. P. Chimento, A. S. Jakubi, and D. Pavon, "Enlarged quintessence cosmology," Physical Review D, vol. 62, Article ID 063508, 2000.

[25] L. P. Chimento, A. S. Jakubi, and D. Pavon, "Dark energy, dissipation, and the coincidence problem," Physical Review D, vol. 67, Article ID 087302, 2003.

[26] M. U. Farooq, M. Jamil, and U. Debnath, "Dynamics of interacting phantom and quintessence dark energies," Astrophysics and Space Science, vol. 334, no. 2, pp. 243-248, 2011.

[27] C. M. Will, Theory and Experiment in Gravitational Physics, Cambridge University Press, 1993.

[28] C. G. Boehmer, G. Caldera-Cabral, R. Lazkoz, and R. Maartens, "Dynamics of dark energy with a coupling to dark matter," Physical Review D, vol. 78, Article ID 023505, 2008.

[29] L. Amendola and D. Tocchini-Valentini, "Stationary dark energy: the present universe as a global attractor," Physical Review D, vol. 64, no. 4, Article ID 043509, 5 pages, 2001.

[30] P. A. R. Ade, N. Aghanim, C. Armitage-Caplan et al., "Planck 2013 results. XVI. Cosmological parameters," http://arxiv.org/ abs/1303.5076.

[31] L. N. Granda, "Transporte de señales eléctricas en microtúbulos: una aproximación cuántica," Revista Colombiana de Física, vol. 42, no. 2, pp. 63-251, 2010.

[32] V. Faraoni, "Inflation and quintessence with nonminimal coupling," Physical Review D, vol. 62, Article ID 023504, 2000.

[33] V. Sahni, T. D. Saini, A. A. Starobinsky, and U. Alam, "Statefinder-a new geometrical diagnostic of dark energy," Journal of Experimental and Theoretical Physics Letters, vol. 77, no. 5, pp. 201-206, 2003.

[34] U. Alam, V. Sahni, T. D. Saini, and A. A. Starobinsky, "Exploring the expanding Universe and dark energy using the statefinder diagnostic," Monthly Notices of the Royal Astronomical Society, vol. 344, no. 4, pp. 1057-1074, 2003. 

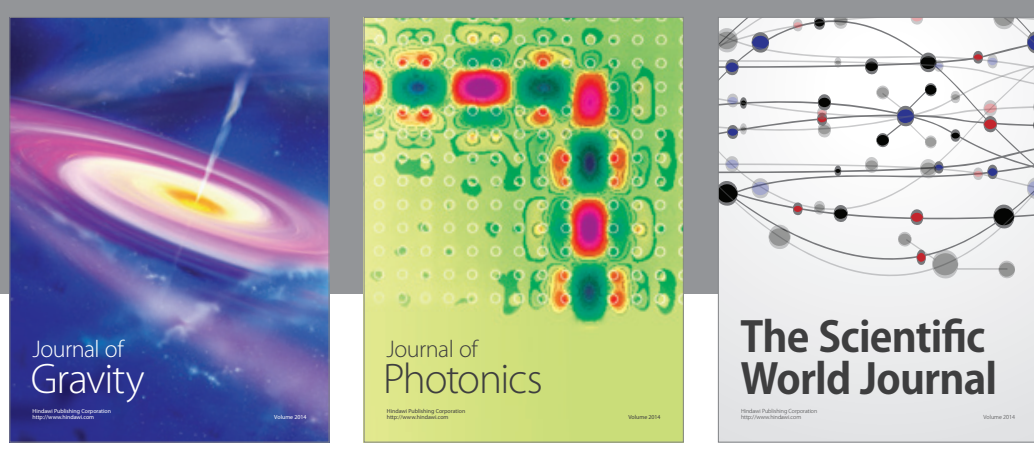

The Scientific World Journal
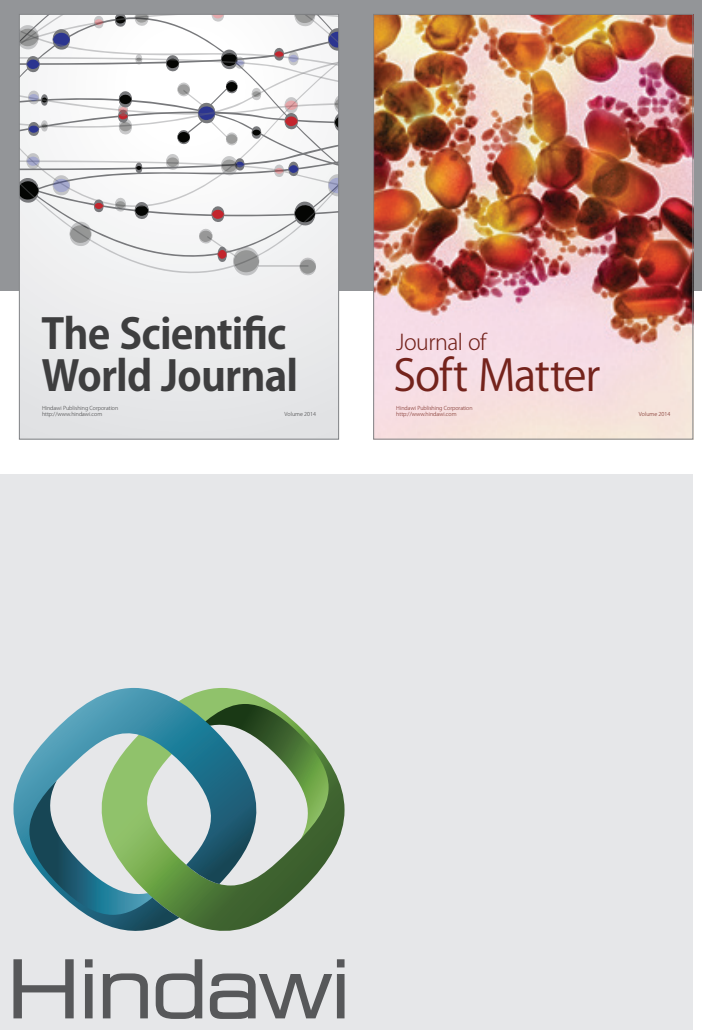

Submit your manuscripts at

http://www.hindawi.com

nternational Journal of

Statistical Mechanics
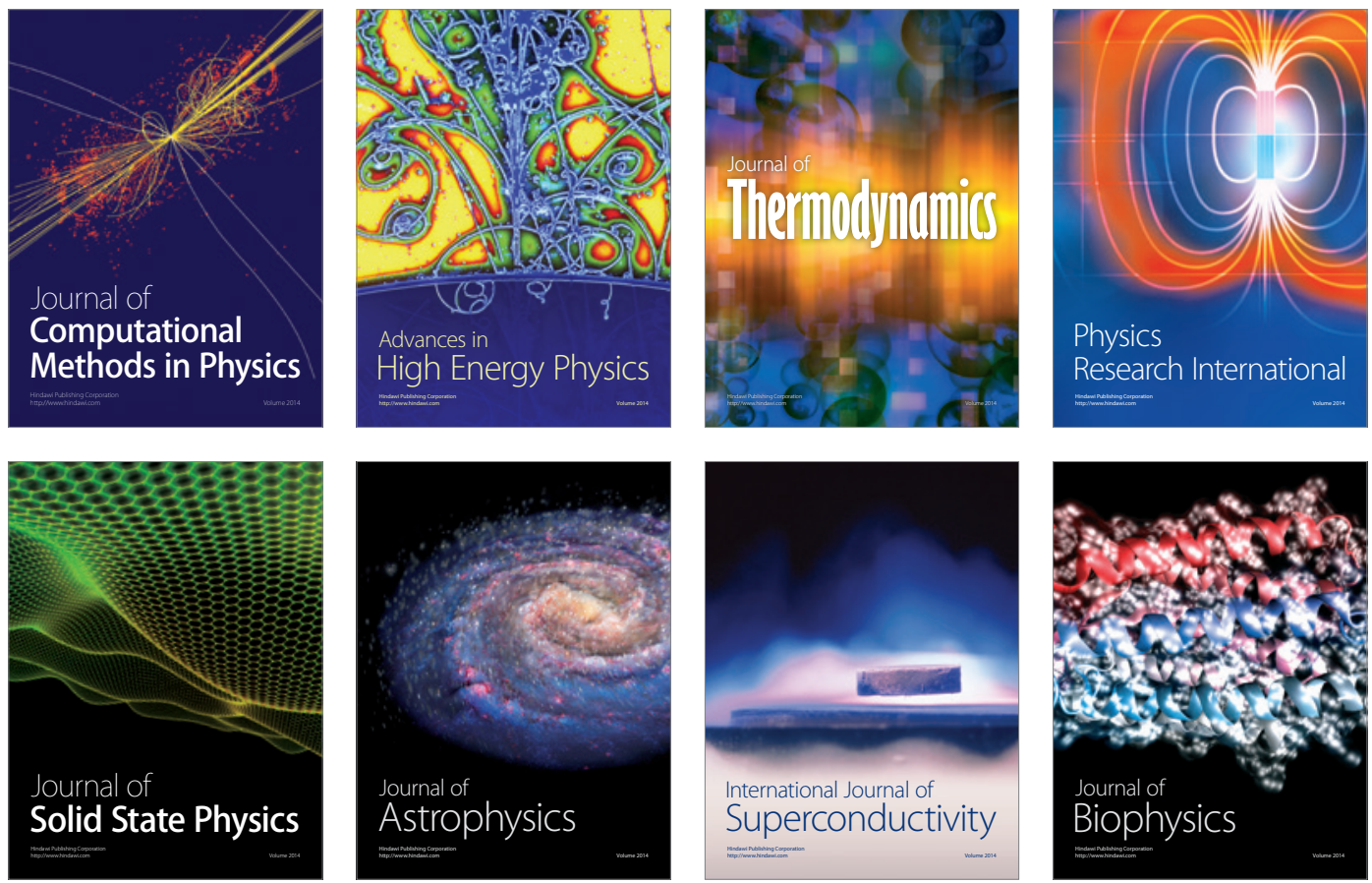
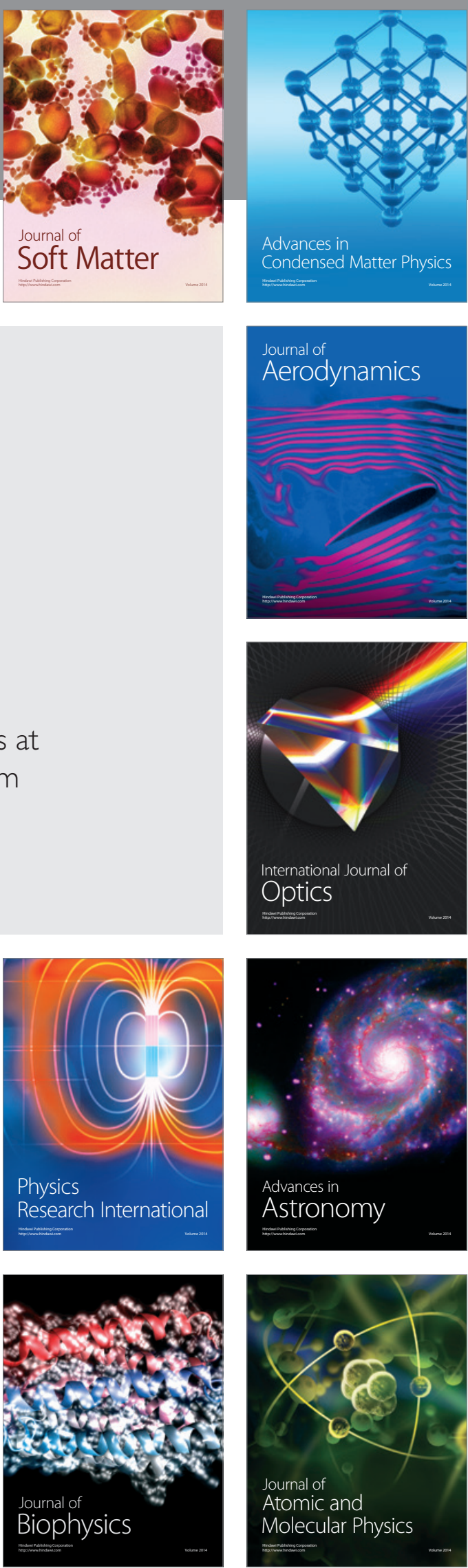